This is the postprint version of the following article: Hanske C, Sanz-Ortiz MN, Liz-Marzán LM. SilicaCoated Plasmonic Metal Nanoparticles in Action. Advanced Materials. 2018:1707003. doi: 10.1002/adma.201707003...This article may be used for non-commercial purposes in accordance with Wiley Terms and Conditions for Self-Archiving. 
DOI: $10.1002 /$

Article type: Review

\section{Silica-Coated Plasmonic Metal Nanoparticles in Action}

Christoph Hanske, *广 Marta N. Sanz-Ortiz, ${ }^{\Perp}$ and Luis M. Liz-Marzán ${ }^{* 广 \$}$

Dr. C. Hanske, Prof. Dr. L. M. Liz-Marzán

† CIC biomaGUNE and CIBER-BBN, Paseo de Miramón 182, 20014 Donostia - San

Sebastián, Spain

$\S$ Ikerbasque, Basque Foundation for Science, 48013 Bilbao, Spain

Email: cmhanske@cicbiomagune.es; 1lizmarzan@cicbiomagune.es

Dr. M. N. Sanz-Ortiz

$\Perp$ Centre for Nanostructured Media, School of Mathematics and Physics, Queen's University Belfast, Belfast BT7 1NN, UK

Keywords: core-shell colloids, plasmonic sensing, theranostics, nanoparticle catalysis, plasmon-enhanced solar cells

Hybrid colloids consisting of noble metal cores and metal oxide shells have been under intense investigation for over two decades and have driven progress in diverse research lines including sensing, medicine, catalysis, and photovoltaics. Consequently, plasmonic core-shell particles have come to play a vital role in a plethora of applications. We provide in this review an overview of recent developments in the design and utilization of the most successful class of such hybrid materials: silica-coated plasmonic metal nanoparticles. Besides summarizing common simple approaches to silica shell growth, special emphasis is put on advanced synthesis routes that either overcome typical limitations of classical methods, such as stability issues and undefined silica porosity, or grant access to particularly sophisticated nanostructures. We hereby describe how different types of silica can be used to provide noble metal particles with specific functionalities and review applications of such nanocomposites in ultrasensitive analyte detection, theranostics, catalysts, and thin film solar cells.

\section{Introduction}

Noble metal/silica composites are both ancient and modern materials, whose exceptional stability has been confirmed by sophisticated artwork like the fourth century Lycurgus cup, as much as by comparatively simple stained glass windows exhibiting lively colors even after weathering for centuries. Responsible for some of the permanent, bleach-resistant colors of stained glasses are localized surface plasmons, i.e., coherent oscillations of the conduction electrons along the surfaces of embedded noble metal nanoparticles (NPs), which are excited by resonant electromagnetic waves. ${ }^{[1]}$ The frequency of localized surface plasmon resonance 


\section{WILEY-VCH}

(LSPR) modes depends on the nanoparticle composition, as well as on their size, shape and the surrounding environment. ${ }^{[2]}$ In the context of modern 'craftsmanship', the synthesis and application of individual plasmonic colloids encapsulated in silica shells or, more general, metal oxide shells with thicknesses ranging from few nanometers to potentially over a hundred nanometers, have attracted tremendous interest during the last two decades. ${ }^{[3]}$ The reason for the vast amount of research dedicated to the development of such nanohybrids is the plain and simple fact that bare metal nanoparticles are poorly suited for many practical applications: while a wide range of scalable synthetic methods are available for the preparation of well-defined plasmonic nanostructures such as spheres $,{ }^{[4,5]} \operatorname{rods},{ }^{[6,7]}$ triangles, ${ }^{[8]}$ stars, ${ }^{[9]}$ cubes, ${ }^{[10]}$ or polyhedra, ${ }^{[11]}$ the harsh conditions required for many applications can severely undermine all efforts to tailor particle properties, inducing aggregation, etching, or other undesired effects. To improve the manipulation of plasmonic particles and facilitate their integration into sensors, photovoltaics cells, catalysts or other functional devices, coating with rigid shells of a chemically inert material such as silica has become a prominent approach. ${ }^{[12,13]}$ Besides the widespread Stöber synthesis of dense silica shells, more recent developments allow us to incorporate typical mesoporous materials such as MCM-41, to a high level of precision and reproducibility. ${ }^{[14,15]}$ As a consequence, research in the field of metal/silica hybrids has recently witnessed a topical shift, from the development of new synthetic protocols toward a smart utilization of core-shell particles and hybrids for novel applications.

In the context of the $30^{\text {th }}$ anniversary of Advanced Materials, we find that an updated review of plasmonic/silica hybrids and their introduction in practical applications is most appropriate. Therefore, this review is organized in three main sections: we first present a systematic categorization of the functionalities of silica shells in the context of their application fields. Thereafter we provide a brief overview of the established synthesis strategies for noble metal/silica hybrid particles, including both dense and mesoporous shells, and we highlight some recently developed, less common preparation methods as well as examples of more 


\section{WILEY-VCH}

complex nanostructures such as Janus particles and their assemblies. Finally, what we consider the most important practical applications of plasmonic nanocomposites are discussed in detail, explaining the rationale behind typically employed designs and suggesting possible directions for further research.

\section{Purposes of Silica Shells}

Most of the synthesis strategies for noble metal/silica composites presented in this article have been refined and extended to allow for a relatively straightforward exchange of the core particles. However, tailoring the specific features of the silica shells such as thickness, condensation degree, or porosity, toward the requirements of a specific application is just as essential as the choice of the core. We aim to provide in this section an overview of various objectives that can be fulfilled by silica shells, as illustrated in Figure 1. These can be categorized into four main motivations for encapsulating metal nanoparticles with silica (or other metal oxides), namely: stabilization of the cores (Figure 1a-c), insulation from the surrounding medium (Figure 1d,e), templating of novel structures (Figure 1f), and controlling molecular transport through the pores (Figure 1g,h). Each of these categories is discussed in a separate section of this chapter, providing an overview of those applications that rely on specific functionalities and summarizing the most relevant literature. 

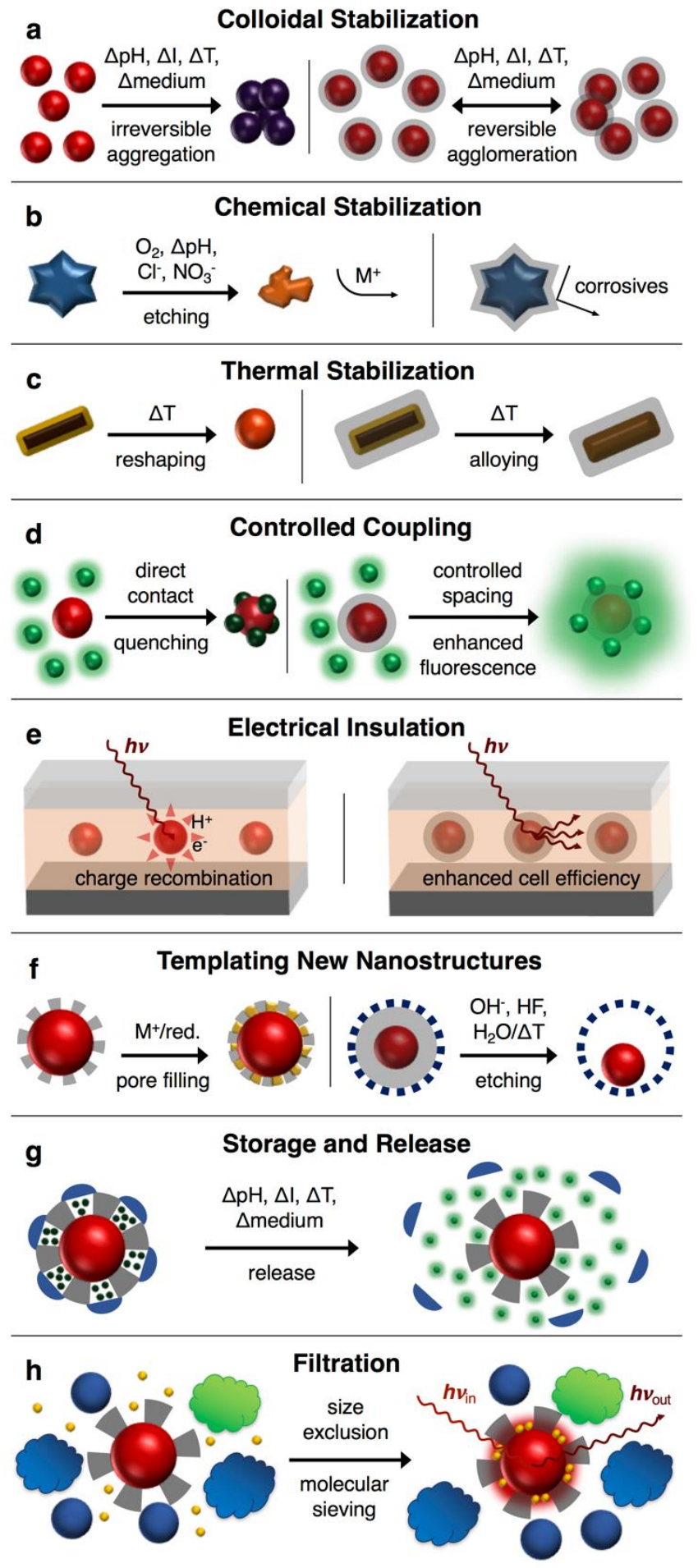

Figure 1. Typical functions of dense and porous silica shells (illustrated for gold nanoparticles of different shapes with their corresponding typical colors). a) Silica coating can be used to stabilize particles that may undergo aggregation and variation of their optical properties upon changes in the $\mathrm{pH}$, ionic strength, temperature or solvent composition. b) Dissolution of metal particles in oxidizing media can be efficiently retarded by a dense, chemically inert silica shell. c) Thermal reshaping is suppressed due to added mechanical stability in the presence of a rigid silica shell. d) Spacing with nanometer precision can be achieved with silica layers, providing control over optical coupling phenomena. For example, luminescence quenching upon direct contact between quantum dots and plasmonic particles (left) can be turned into fluorescence enhancement by a spacer of optimized thickness (right). e) Electrical insulation with $\mathrm{SiO}_{2}$ 


\section{WILEY-VCH}

prevents unwanted charge carrier recombination at metal surfaces (left) enabling the integration of power conversion enhancing plasmonic nanoparticles into thin film solar cells (right). f) Silica-assisted templating of unique nanostructures is facilitated by filling the pores of a mesoporous shell via surface-catalyzed metal ion reduction (left), or by dissolution of a sacrificial $\mathrm{SiO}_{2}$ layer (right) leading to yolk-shell morphologies. g) Storage of an active compound, e.g. a drug, inside silica mesopores in combination with triggered release mechanisms is the principle of therapeutic nanoparticle systems aiming at efficient treatment with reduced side effects. h) Mesoporous shells may also enable size-selective uptake of small molecules from complex media and sensitive detection by sensing methods that rely on close contact between the analyte and a plasmonic nanostructure.

\subsection{Colloidal, Chemical and Thermal Stabilization}

Ensuring colloidal stabilization against aggregation and particle fusion, as illustrated in Figure

1a-c, has been the prime motivation behind the development of a complete toolbox of methods for coating noble metal nanoparticles with silica. The stabilizing effect of silica shells is attributable to two main effects: ${ }^{[13]}$ due to the exposed silanol groups, cations readily accumulate near silica surfaces, leading to long range electrostatic repulsion forces between the particles. At small interparticle distances, additional steric repulsion arises because of the comparatively low Hamaker constant of silica (in water $A_{\text {silica }}=1 \times 10^{-20} \mathrm{~J}$ versus $A_{A u}=4 \times 10^{-19} \mathrm{~J}$ ) and the consequently weaker van der Waals interactions. ${ }^{[16]}$ The resulting electrosteric stabilization facilitates a transfer of metal nanoparticles with sufficiently thick silica coatings into aqueous or organic media and has been shown to efficiently prevent irreversible aggregation, even in liquids with extremely high ionic strengths such as simulated body fluids and typical saline buffers. ${ }^{[17,18]}$ Besides its key importance for biomedical usage, high colloidal stability is also advantageous for applications in nanophotonics and catalysis, as aggregation and eventual fusion of metal nanoparticles is known to induce drastic changes in the plasmonic properties and a significant loss of activity, in particular after high temperature treatments. ${ }^{[19-}$ ${ }^{23]}$ Moreover, the improved mechanical stability imposed by the coating shells can be used to prevent morphological changes during the controlled alloying of bimetallic particles, ${ }^{[24,25]}$ and has been employed to fixate the shape of $3 \mathrm{D}$ nanoparticle clusters. ${ }^{[26-28]}$ Besides effectively inhibiting the oxidative etching of less noble metals such as silver or copper, as well as the 


\section{WILEY-VCH}

leakage of toxic ions from quantum dots (QDs), encapsulation in dense silica shells offers excellent means to incorporate and protect fluorescent or Raman-active dye tags. ${ }^{\text {[29-32] }}$

\subsection{Precision Spacing and Insulation}

Coating plasmonic nanoparticles with silica usually causes a red shift of the LSPR due to a local increase of the refractive index, as well as a redistribution of the electric near-field, which becomes progressively concentrated within the silica layer when the shell thickness is enlarged. ${ }^{[33]}$ Excellent control over the shell dimensions can be readily achieved for layers thicker than $2 \mathrm{~nm}$, leading to accurate tuning of the optical properties of separated, non-coupling composite particles. Apart from facilitating the systematic investigation of the fundamental optical properties of isolated plasmonic nanocrystals by providing a well-defined dielectric environment, ${ }^{[34-36]}$ precise spacing is paramount for tuning light absorption and scattering properties of particle clusters and heterodimers due to interparticle coupling, as well as for controlling the transfer of energy from metallic cores to nearby fluorescent materials (Figure 1d). ${ }^{[37-39]}$ Practical applications that have largely profited from the high precision of silica coating methods include metal-enhanced fluorescent emitters and sensors based on refractive index changes. ${ }^{[40-44]}$ Another representative example is the use of shell-isolated nanoparticles (SHINs) to monitor reaction kinetics. In particular, SHIN-enhanced Raman spectroscopy (SHINERS) relies on the use of ultra-thin, dense silica shells to suppress the interference of the metal surface with the investigated chemical reaction, thereby allowing an undisturbed spectroscopic characterization via surface-enhanced Raman scattering (SERS) spectroscopy. ${ }^{[45-}$

${ }^{47]}$ On the other hand, encapsulation of noble metal nanoparticles within silica shells of only a few $\mathrm{nm}$ thickness has become a standard procedure in the fabrication of plasmonically enhanced solar cells, whose efficiency has been shown to strongly depend on electric insulation and the ability to concentrate optical near-fields at the surrounding matrix (Figure 1e). ${ }^{[48-53]}$ 


\section{WILEY-VCH}

\subsection{Templating Complex Nanostructures}

The affinity of metal surfaces for silica (vitreophilicity) has traditionally been a hurdle to overcome for efficient metal particle encapsulation. ${ }^{[54]}$ This can however be used as an advantage, as both site-selective vitreophilization of metal nanoparticles, and controlled shell porosity may lead to the construction of complex nanostructures such as Janus bimetallic heteroparticles, branched composites, or yolk-shell structures (Figure 1f). ${ }^{[14,55-59]}$ However, rather than increasing complexity, for many practical applications a seemingly trivial property of silica shells is a key advantage: robust and straightforward surface functionalization via silane coupling chemistry. Whereas hydrophobation is necessary for certain self-assembly approaches, ${ }^{[60,61]}$ establishing long-lasting biocompatibility is crucial for the successful application of plasmonic particles in biotechnology. It has been shown that silica coating can become a reliable method to reduce toxicity, since it avoids the use of weakly bound ligands and prevents leakage of ions from the cores. ${ }^{[30,62]}$ On the other hand, the outmost silanol groups allow straightforward attachment of targeting moieties, thus providing a basis for plasmonic bioimaging and theranostic applications. ${ }^{[63-66]}$

\subsection{Encapsulation and Filtration}

Nanoprobes for bioassays and theranostics generally rely on the integration of active molecules or nanoparticles inside the noble metal/silica composites. Besides metal-enhanced fluorescence, SERS based methods in which the core particles provide near-field enhancement of molecular tags, as well as multi-modal combinations with other methods, have attracted much interest. The growth of silica shells facilitates the fabrication of nanoprobes where tags can be directly integrated within the silica network. ${ }^{[28,67,68]}$ Moreover, the high specific area of mesoporous silica is frequently exploited to increase the loading with small signal molecules or drugs, in such a way that the pores can be closed with trigger-sensitive gates to control subsequent release at the site of action (Figure 1g). ${ }^{[27,69-75]}$ Thereby, the pores not only temporally retain and 


\section{WILEY-VCH}

passivate the drugs, but also protect them for a greater chemical stability. Apart from drug release, the pores may also serve as a molecular sieve to enhance the selectivity toward ions and small analyte molecules in complex biological media, as illustrated in Figure 1h. ${ }^{[14,76-80]}$

\section{Synthesis of Noble Metal/Silica Hybrid Particles}

Since the first reports on gold/silica hybrid nanoparticles were published over two decades

ago, ${ }^{[81-83]}$ a plethora of protocols for core-shell particle synthesis have been devised, granting access to almost every imaginable noble metal/metal oxide combination, as well as to many different morphologies. ${ }^{[3]}$ Instead of recapturing in detail the manifold developments in this mature field, we restrict ourselves to a brief overview of the well-established synthesis strategies for the growth of both dense and porous silica shells. For a comprehensive survey of the available procedures the reader is kindly referred to recent review articles. ${ }^{[3,12,13,59]} \mathrm{We}$ additionally include a selection of rather unconventional methods that either yield complex nanostructures, show exceptional potential for upscaling, or simply report on unorthodox but noteworthy approaches to noble metal/silica composite synthesis.

\subsection{Silica Growth on Nanoparticles}

Seeded growth methods, in which pre-synthesized noble metal nanoparticles are overcoated with silica by means of a modified Stöber process, represent by far the most common route toward plasmonic core-shell nanostructures. ${ }^{\left[{ }^{84,85]}\right.}$ Hereby, the metal particles act as nucleation centers for the condensation of silicic acids, typically produced via hydrolysis and condensation of tetraethylorthosilicate (TEOS) in an alkaline alcohol/water mixture. Whereas this two-step procedure offers flexibility regarding the shape of the core particles, as well as easy adjustment of the shell thickness through the seed/TEOS ratio, it usually requires functionalization/priming of the nanoparticles surface with certain ligands, for two main reasons. First, for practically all combinations of noble metal surfaces and metal oxides, phase separation into heteroparticles 


\section{WILEY-VCH}

minimizing the interfacial area between the two components is energetically favorable as compared to homogeneous overgrowth. ${ }^{[59]}$ Second, both particles that are synthesized in nonpolar solvents (carrying hydrophobic ligands) and those prepared in water (commonly stabilized by surfactants), may lack colloidal stability in the standard Stöber reaction medium, leading to aggregation issues, especially during the early stage of silica shell growth. ${ }^{[13]}$

\subsection{Dense Silica Shells}

To alleviate these issues, nanoparticles are commonly covered by small, covalently binding molecules or polymers that decrease the interfacial energy between the metal and silica, if necessary providing additional (electro-)steric stabilization. For the vitreophilization of $\mathrm{Au}, \mathrm{Ag}$, Pt and other noble metals, ligands containing both thiols or amines (which readily bind to metal surfaces) and hydrolysable alkoxysilane groups or terminal acid moieties have been frequently used. ${ }^{[85-88]}$ Typical examples of such low molecular weight surface primers are amino- and mercaptosilanes, ${ }^{[39,85,89]}$ mercaptocarboxylic acids, ${ }^{[56,87,90-93]}$ or mercaptoethanesulfonate. ${ }^{[78]}$ In cases of serious aggregation issues, polymeric stabilizers are regarded advantageous due to their ability to strongly attach to the metal surface via multiple binding sites adding permanent (electro-)steric stabilization. Introduced by Graf et al. as a universal coating procedure, ${ }^{[83]}$ capping nanoparticles with poly-(N-vinylpyrrolidone) (PVP), an amphiphilic, non-ionic polymer often used in the preparation of spiky and anisotropic Au or Ag nanoparticles, ${ }^{[94,95]}$ has become widely adopted for the fabrication of noble metal/silica composites. Apart from its ability to stabilize a wide range of inorganic and polymeric nanoparticles toward silica coating, PVP has also been demonstrated to permit the growth of other metal oxide shells like $\mathrm{ZnO}, \mathrm{TiO}_{2}$, $\mathrm{Co}_{2} \mathrm{O}_{3}, \mathrm{Fe}_{3} \mathrm{O}_{4}, \mathrm{Gd}_{2} \mathrm{O}_{3}$ when used in conjunction with covalently binding, hydrophilic ligands like 4-mercaptoundecanoic acid. ${ }^{[96]}$ Besides PVP, thiol-modified poly(ethylene glycol), and polyelectrolyte multilayers made by layer-by-layer (LbL) assembly, have been successfully used as stabilizing interlayers between noble metal particles and silica, even giving access to 


\section{WILEY-VCH}

more complex particle morphologies by integration of molecular tags, luminescent moieties, or paramagnetic nanoparticles. ${ }^{[60,67,97-99]}$

An alternative to coating in standard Stöber media comprises conducting silica condensation in water-in-oil microemulsions, where the precursor TEOS is dissolved in the oil phase, while the water droplets harbor the hydrophilic, vitreophilic cores. ${ }^{[100]}$ Emulsification is achieved by sonication of oil-water mixtures, in the presence of a surfactant such as cetyltrimethylammonimum bromide (CTAB), sodium dioctylsulfosuccinate (AOT), or polyoxyethylene(5)nonylphenylether (Igepal ${ }^{\circledR}$ CO-520). ${ }^{[101,102]}$ A particular appeal of this technique lies in the possibility of synthesizing both the cores and the silica shells in a one-pot reaction medium, as demonstrated for core-shell particles as well as for yolk-shell structures, in which the plasmonic cores are detached from the silica layer. ${ }^{[103,104]}$

\subsection{Mesoporous Silica Shells}

A variety of applications, including drug delivery or nanoparticle catalysis, require coatings that allow some degree of contact between the cores and the solvent, and in such cases mesoporous silica is preferred instead of dense, compact shells. Molecular diffusion through the weakly crosslinked silica network is an intrinsic property of nanometer thin silica shells produced by polymerization of sodium silicate. This was demonstrated by Mulvaney and coworkers by monitoring both chemical reactions on the metal cores within an electron microscope ${ }^{[29]}$ and the spectral shift of encapsulated $\mathrm{Ag}$ nanoparticles due to electron injection induced by $\mathrm{NaBH}_{4}$ in solution. ${ }^{[105]}$ Porosity can be introduced in thicker shells, either postsynthetically by etching in an alkaline medium, but also facilitated during the shell growth by incorporating organic porogens such as n-octadecyl trimethoxysilane (TMS), or templated by silica condensation in the presence of micelles of surfactants like TTAB or CTAB. ${ }^{[14,15,22,106]}$ These micellar scaffolds direct the deposition of silica via the distribution of their hydrophilic and hydrophobic regions, with a precise definition of pore size and morphology. The first 


\section{WILEY-VCH}

successful silica coating on $\mathrm{CTAB}$ stabilized particles without further vitreophilization was demonstrated by Gorelikov and Matsuura, who reported the formation of shells with uniform thickness and disordered pores of about $4 \mathrm{~nm}$ diameter, by conducting the Stöber process in water at room temperature. ${ }^{[107]}$ Interestingly, radially oriented pores with diameters of approximately $2 \mathrm{~nm}$ can also be obtained, by growing the shells at $60{ }^{\circ} \mathrm{C}$ in water/ethanol mixtures. ${ }^{[14]}$ In a similar procedure, precise control over the shell thickness was achieved in the range of 3-17 nm by slow addition of TEOS using a syringe pump, and regular shells as thin as $2 \mathrm{~nm}$ were obtained by quenching silica condensation with PEG-silane after a certain reaction time. ${ }^{[108]}$ To open the pores, residual surfactants can either be removed by calcination or, in the case of the commonly employed $\mathrm{CTAB}$, washed out by dilute $\mathrm{HCl}$ or ammonium nitrate solution. ${ }^{[109]}$ An illustration of a complete synthesis route toward gold nanoparticles (AuNPs) coated with mesoporous silica, including all steps from the initial precursor reduction to the final pore cleaning step, is presented in Figure 2.

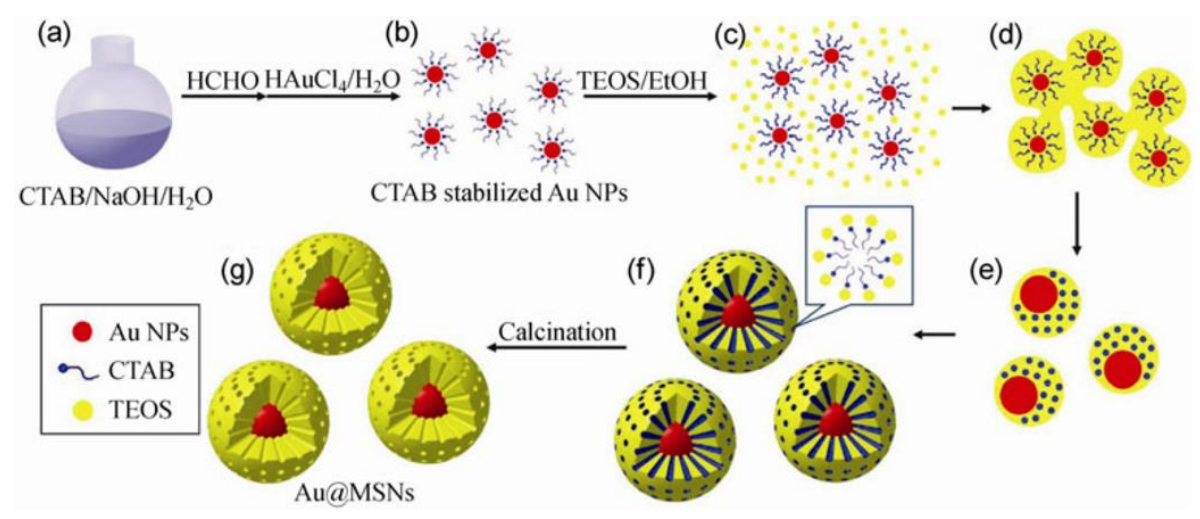

Figure 2. Schematic illustration of a one-pot synthesis of $\mathrm{Au} / \mathrm{mSiO}_{2}$ nanoparticles. a) Core synthesis via reduction of a precursor gold salt. b) Transfer of CTAB stabilized AuNPs into a Stöber reaction medium. c-e) Growth of mesoporous silica shells by hydrolysis and condensation of TEOS in the presence of the micellar CTAB template. f) Removal of pore filling CTAB by calcination. g) Final morphology with plasmonic cores accessible to small molecules through open pores. Reproduced with permission. ${ }^{[15]}$ Copyright 2013, SpringerVerlag.

One important requirement applying to all of these synthesis methods is a tight control of the silica condensation kinetics, which is achieved by careful adjustment of the $\mathrm{pH}$, temperature, 


\section{WILEY-VCH}

solvent composition, and precursor to nuclei ratio. Failure to balance these parameters can lead to aggregation of the cores or homogeneous nucleation of empty silica particles.

\subsection{Unconventional Routes}

Silica shells prepared by the different sol-gel approaches presented in the previous section are usually characterized by a low condensation degree and a high content of silanol $(\mathrm{Si}-\mathrm{OH})$ groups, which reduce their chemical stability in water and biological media. ${ }^{[17]}$ In basic environments or even in pure water, silanol groups in the silica network can readily lead to dissolution of the shells on time scales ranging from minutes to days. ${ }^{[88]}$ The most extended method to improve the stability of silica in water, producing particles that retain their properties over months, is a temperature treatment that may involve heating the sample for several hours in the $100{ }^{\circ} \mathrm{C}-600{ }^{\circ} \mathrm{C}$ range, or subjecting it to extended reflux in boiling solvents. ${ }^{[14,110]}$ However, depending on the targeted application, this process might present different drawbacks. The temperature treatment tends to make the silica shell more brittle and, in the case of mesoporous silica, can cause reshaping of the pore geometry. ${ }^{[111]}$ It also causes the loss of surface silanol groups that might be required for further functionalization of the particles via silanization. Moreover, in the case of silica-coated noble metal nanoparticles a high increase in temperature may lead to reshaping of anisotropic nanoparticles. ${ }^{[14]}$ In this section, we present examples of coating methods that either focus on improving problematic aspects of Stöberbased silica, such as low hydrolysis resistance or slow reaction progression, or pave the way to completely new, complex nanostructures.

An interesting way to improve silica stability in water was recently reported by Montaño-Priede et al., who developed an ultrasound-assisted variation of the Stöber process (see Figure 3), which yields silica shells with enhanced hydrothermal stability. ${ }^{[17]}$ For this purpose, gold nanospheres functionalized with methoxy-polyethyleneglycol thiol (mPEG-SH) were overgrown with 6-10 nm thick silica shells, under sonication. The core-shell particles prepared 


\section{WILEY-VCH}

by this method displayed significantly improved stability during hydrothermal treatment at

$90{ }^{\circ} \mathrm{C}$ and high resistance against agglomeration in a simulated body fluid. This was attributed

to a reduced amount of silanol groups in the silica network due to an acceleration of the TEOS

hydrolysis and condensation reactions by ultrasound.
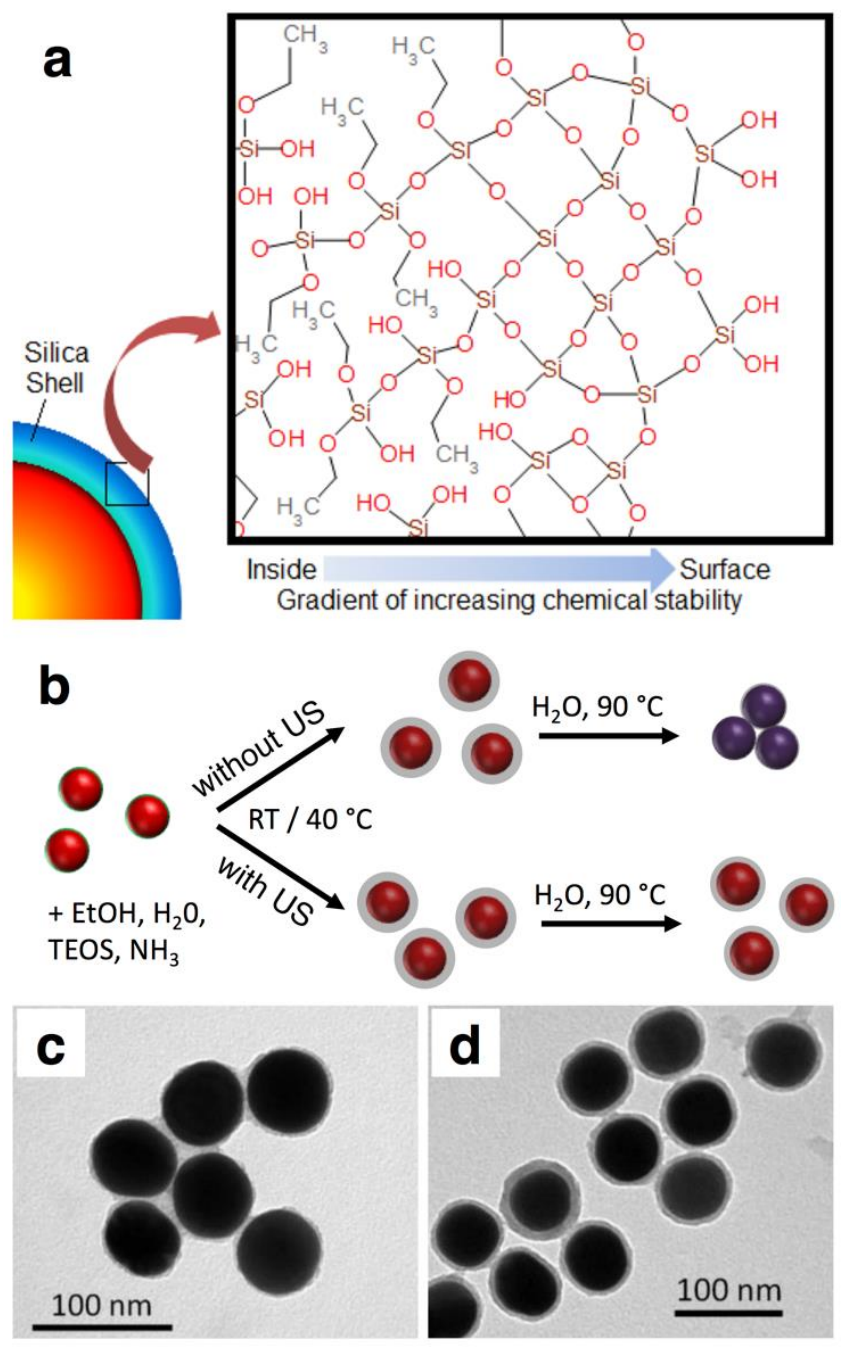

Figure 3. Ultrasound-assisted synthesis of $\mathrm{Au} / \mathrm{SiO}_{2}$ particles with chemically stable silica shells. a) Scheme of a silica-coated nanoparticle showing the chemical inhomogeneity of a silica network typically obtained by the Stöber process. b) Schematic representation of conventional and ultrasound-assisted silica coating of PEG-SH functionalized nanoparticles and the result after hydrothermal etching. c,d) TEM micrographs of $\mathrm{Au} / \mathrm{SiO}_{2}$ particles made by regular (left) and ultrasound-assisted (right) silica coating after $24 \mathrm{~h}$ of hydrothermal treatment at $90{ }^{\circ} \mathrm{C}$ : sonication during hydrolysis and condensation of the precursor significantly increased the shell stability, thereby preventing irreversible aggregation in water. Adapted with permission. ${ }^{[17]}$ Copyright 2017, American Chemical Society.

Resistance against aggregation and dissolution in biological media gains critical importance when the bare core material displays high toxicity. A frequently discussed example is the metal 


\section{WILEY-VCH}

silver, which has excellent plasmonic properties but is often excluded from biosensing applications due to its known cytotoxicity. An interesting approach to fabricate hermetically encapsulated silver nanoparticles was demonstrated by Sotiriou et al., who performed the dry synthesis of Ag nanoparticles by a flame aerosol method, followed by swirl injection of a silica precursor. ${ }^{[30]}$ The efficacy of the encapsulation procedure was probed by investigating the impact of the particles on E. coli bacteria, while the thin shell still allowed the detection of bovine serum albumin via LSPR sensing. Whereas flame spray pyrolysis yields particles of a much broader size distribution than the usual wet chemical seeded growth methods, this was shown to be a readily scalable, continuous process, which grants access to particles with closed silica shells as thin as 1 nm. ${ }^{[112]}$

A high-speed coating procedure yielding monodisperse $\mathrm{Ag} / \mathrm{SiO}_{2}$ nanoparticles was demonstrated by Bahadur et al. ${ }^{[113]}$ Citrate-capped $17 \mathrm{~nm}$ silver spheres were directly encapsulated without preceding ligand exchange by microwaving a mixture of metal cores, TEOS, and dimethylamine under reflux for just 2 minutes. The shell thickness could be adjusted over a range of 3-80 nm, by simply varying the concentration of TEOS, without inducing aggregation of the core particles. For encapsulation of ultrasmall particles with diameters of few nanometers, conventional Stöber synthesis and micro-emulsion encapsulation are regarded problematic because of difficulties in efficiently binding the core surfaces to silica. ${ }^{[114]}$ Zhang et al. faced this issue and developed a synthetic route to obtain $\mathrm{Au} / \mathrm{SiO}_{2}$ particles, in which gold hydroxide nanoparticles, rather than the metal, were encapsulated in inverse micelles by in situ formation and subsequent silica condensation, followed by thermal annealing that resulted in reduction of the cores and formation of the desired metal/silica composites with core diameters between 1.5 and $2.3 \mathrm{~nm}$ and a shell thickness around $10 \mathrm{~nm} \cdot{ }^{[14]}$ The amorphous shell both effectively stabilized the particles against sintering and displayed sufficient gas permeability to allow the study of size effects of the ultrasmall Au particles on the catalyzed oxidation of CO. 


\section{WILEY-VCH}

\subsection{Synthesis of Complex Hybrid Nanostructures}

Beyond simple core-shell morphologies, significant effort has been spent toward the preparation of highly complex metal/metal oxide hybrids, whereby typical features of silica shells, such as the general applicability to different materials, precise control over thickness and porosity, as well as adjustable hydrothermal stability, have been exploited to direct the formation of novel nanostructures. A remarkable example toward the formation of Janus metal/silicia hybrids involves the partial vitreophilization of metal nanoparticles, so that shell growth is restricted to the vitreophilic surface regions. Chen et al. demonstrated this approach for the case of gold nanoparticles functionalized with a mixture of the ligands 4mercaptophenylacetic acid and polyacrylic acid. ${ }^{[93]}$ Silica overgrowth yielded a high percentage of $\mathrm{Au} / \mathrm{SiO}_{2}$ Janus particles, which was attributed to an efficient phase separation of the ligands on the gold nanospheres (AuNSs) and the lower affinity of silica toward the polymer-coated part. Employing these Janus particles as seeds during the reduction of $\mathrm{AgNO}_{3}$ led to the formation of ternary $\mathrm{Ag} / \mathrm{Au} / \mathrm{SiO}_{2}$ structures. Similar protecting group strategies were also successfully employed for the preparation of $\mathrm{Au} / \mathrm{SiO}_{2}$ Janus nanoparticles with spiky gold protrusions on the non-coated side, as well as for $\mathrm{Au} / \mathrm{SiO}_{2}$ hybrid core-satellite nanostructures and heterodimers functionalized with two separate Raman-active molecules (Figure 4). ${ }^{[28,56]}$ Apart from molecular SERS detection, such designs have been proposed as multiplex SERS tags for bioimaging purposes. 
a

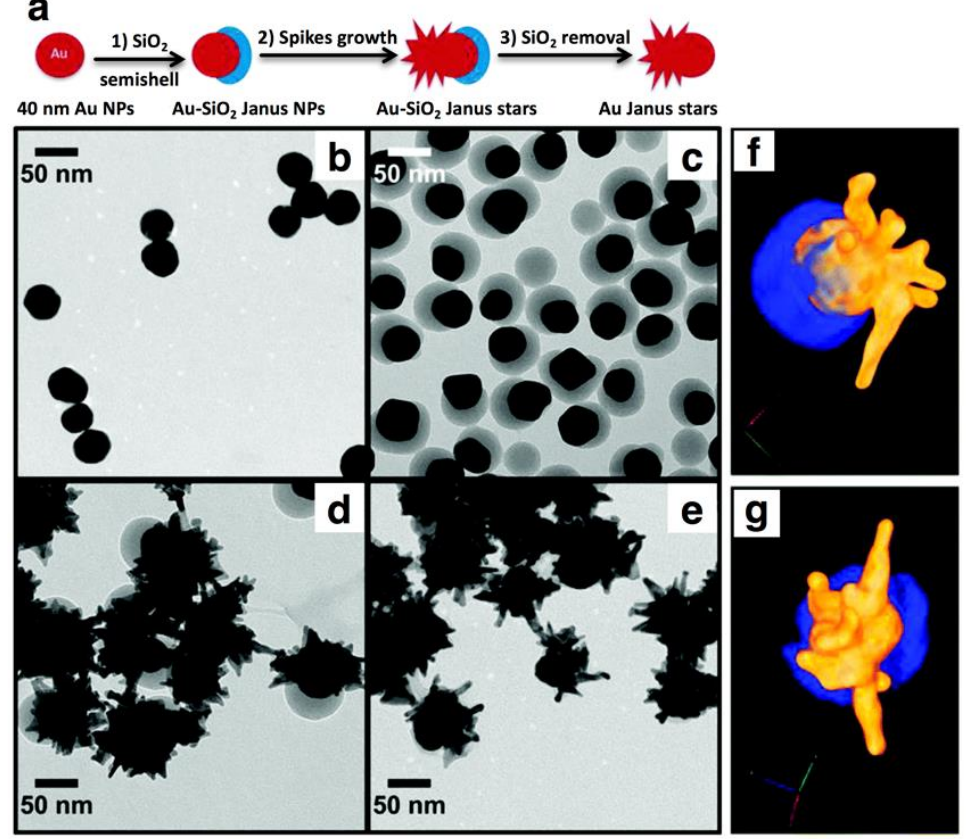

h
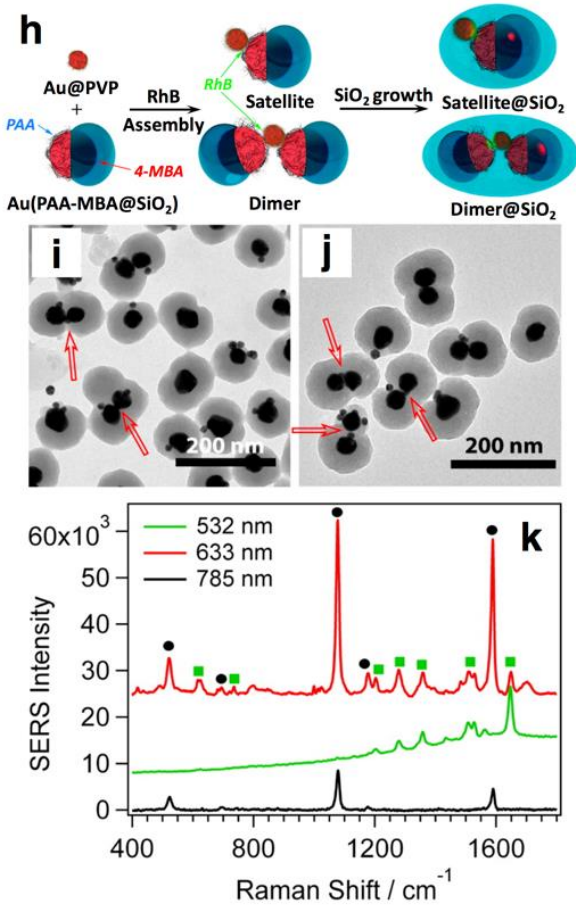

Figure 4. $\mathrm{Au} / \mathrm{SiO}_{2}$ Janus nanostars and $\mathrm{Au} / \mathrm{SiO}_{2}$ core-satellite nanoparticles prepared by silica coating of partially vitreophilized gold cores. a) Schematic representation of local silica patch growth on $\mathrm{Au}$ spheres functionalized with a ligand couple (4-mercaptobenzoic acid (4-MBA) + polyacrylic acid (PAA)) and subsequent metal-catalyzed branching into Janus nanostars. be) TEM images of the depicted synthesis stages: b) $40 \mathrm{~nm} \mathrm{Au}$ spheres, c) $\mathrm{Au} / \mathrm{SiO}_{2} \mathrm{Janus}$ particles, d) $\mathrm{Au} / \mathrm{SiO}_{2}$ Janus nanostars, e) Au Janus nanostars obtained upon dissolution of the silica patches. f,g) HAADF-STEM tomography reconstruction of a $\mathrm{Au} / \mathrm{SiO}_{2} \mathrm{Janus}$ nanostar, shown from two different angles. Adapted with permission. ${ }^{[56]}$ Copyright 2014, Royal Society of Chemistry. h) Scheme of the fabrication of hybrid $\mathrm{Au} / \mathrm{SiO}_{2}$ core-satellite colloids containing two Raman tags: heteroassembly of 4-MBA-containing $\mathrm{Au} / \mathrm{SiO}_{2} \mathrm{Janus}$ particles and $15 \mathrm{~nm}$ spheres in the presence of Rhodamine $B$ Isocyanate $(\mathrm{RhB})$ and further coating with silica. i) TEM images of $\mathrm{Au} / \mathrm{SiO}_{2}$ hybrid satellites. j) Micrographs of dimers with arrows pointing to linking AuNSs. k) SERS spectra of satellite particles recorded in water with $532 \mathrm{~nm}, 633 \mathrm{~nm}$, and $785 \mathrm{~nm}$ lasers: depending on the laser wavelength, RhB (green dots) and 4-MBA signals (black dots) were excited separately or simultaneously, allowing multiplexed SERS bioimaging with a single colloidal system. Adapted with permission. ${ }^{[28]}$ Copyright 2015, American Chemical Society.

A variety of anisotropic, bimetallic particles with potential catalytic applications were demonstrated by Wang et al., who used partial silica coating to template the deposition of Pd, $\mathrm{Pt}$, and Ag onto either the ends or the sides of gold nanorods (AuNRs). ${ }^{[57]}$ As illustrated in Figure 5, the required site-selective silica coating resulted from curvature effects. The lower affinity of CTAB toward the rounded ends of the particles was claimed to be responsible for the preferential deposition of silica at these sites, but also for the apex-specific adsorption of 


\section{WILEY-VCH}

mPEG-thiol, which inhibited silica growth after partial ligand exchange, so that deposition would be favored at the sides. Even though the initial silica patches were mesoporous, the pores could be sealed upon a second silica deposition prior to the final metal overgrowth step. It is worth noting that, as recently shown for gold nanobipyramids (AuNBPs) and gold nanocubes, the efficacy of curvature-induced site-selective silica coating strongly depends on the concentration of the stabilizing surfactant CTAB (Figure 5h-k). ${ }^{[58]}$

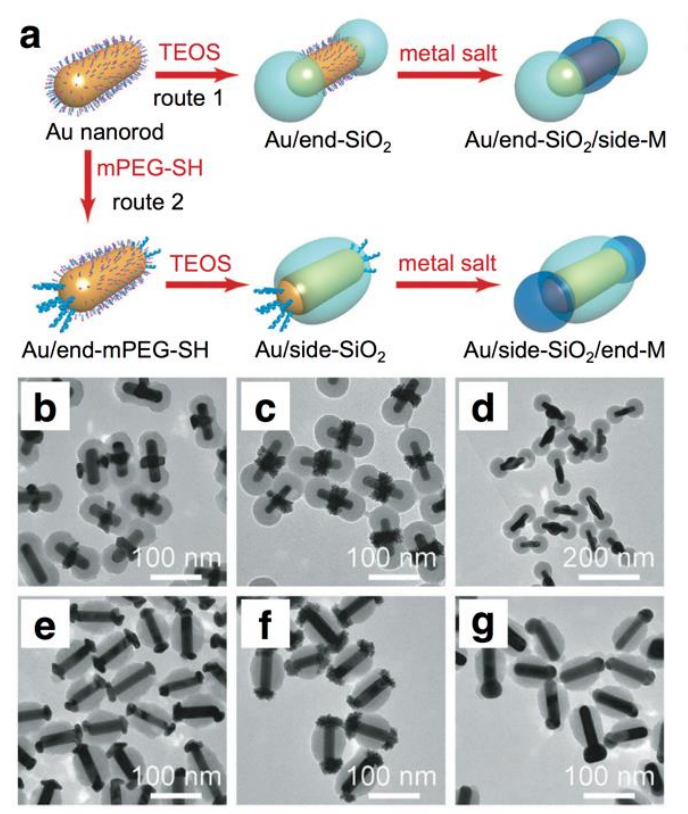

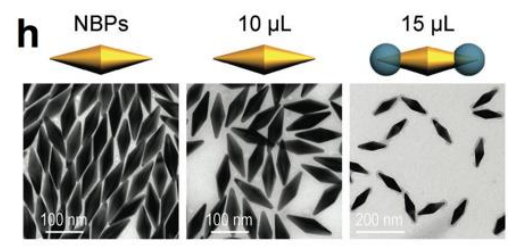
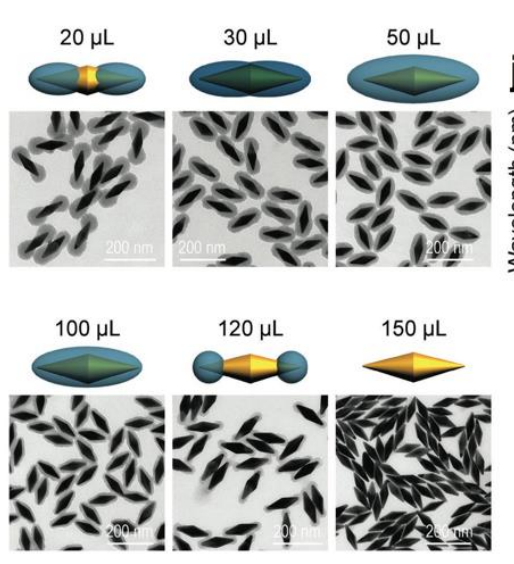
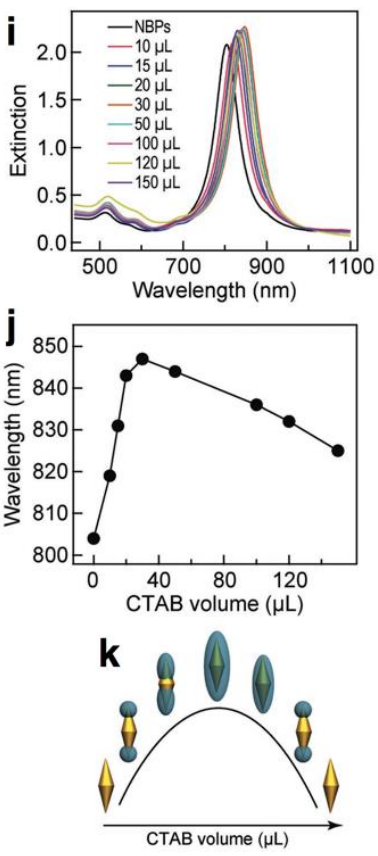

Figure 5. Silica-templated synthesis of anisotropic, bimetallic heterostructures. a) Site selective metal growth on gold nanorods: due to the higher curvature, in route 1 silica growth proceeds preferentially on the ends of CTAB-coated rods enabling subsequent metal deposition on the sides. Prior end-capping with mPEG-SH (route 2) reverses the selectivity. b,c,d) TEM images of silica end-capped AuNRs after overgrowth of Au, Pt, and Ag, respectively. e,f,g) AuNRs with silica-coated sides after overgrowth with $\mathrm{Au}, \mathrm{Pt}$, and $\mathrm{Ag}$, respectively. Metal deposition is catalyzed by the surface of the gold core and occurs only on areas that are not covered by silica. Adapted with permission. ${ }^{[57]}$ Copyright 2013, Wiley-VCH Verlag GmbH \& Co. KGaA, Weinheim. h) Schemes and TEM micrographs of gold nanobipyramids after partial coating with silica in the presence of different amounts of CTAB $(10-150 \mu \mathrm{L}$ of $100 \mathrm{mM}$ CTAB added to NBPs in deionized water). $i, j$ ) Corresponding extinction spectra and LSPR positions for silica coating in the presence of different amounts of CTAB. k) Illustration of CTAB-controlled silica coating: both high and low ligand concentrations prevent silica deposition, whereas intermediate concentrations result in either selective coating of NBP ends or complete encapsulation. Adapted with permission. ${ }^{[58]}$ Copyright 2017, Wiley-VCH GmbH \& Co. KGaA, Weinheim. 


\section{WILEY-VCH}

A different synthetic direction involves the confined metal deposition on a smaller length scale, by using the pores of silica shells themselves as templates. This strategy was introduced for the deposition of noble metals inside the pores of SBA-15 mesoporous silica materials, which feature typical pore diameters of 5-30 nm. ${ }^{[115]}$ Thermal reduction of $\mathrm{Au}, \mathrm{Ag}$, and Pt precursors under $\mathrm{H}_{2}$ flow in the presence of bulk SBA-15 lead to the growth of metal wires inside the hexagonally packed pores. ${ }^{[116]}$ The templating effect of mesopores in metal deposition by wet chemistry was investigated by Angelomé et al. for thin silica films deposited by spin coating of a precursor mixture onto submonolayers of surface adsorbed AuNPs. ${ }^{[117]}$ Through the open pores educts were able to diffuse toward the nanoparticle surfaces, allowing the growth of branches via a metal-catalyzed reduction of $\mathrm{HAuCl}_{4}$, in the presence of $\mathrm{CTAB}$. Varying the pore diameter allowed the fabrication of stable plasmonic films with tailored optical properties, determined by the growth of branches on the existing, spherical nanoparticles. A similar process was later followed by Sanz-Ortiz et al., to grow gold branches and silver shells from the cores of dispersed $\mathrm{Au} / \mathrm{mSiO}_{2}$ particles. Shown in Figure 6 are a selection of particle morphologies, obtained from different $\mathrm{Au}$ nanoparticle cores. By controlling the seed-to-precursor ratio in a surfactant-free metal deposition reaction, the length of the branches could be varied, thereby giving access to stable particles with high SERS enhancement capabilities and a built-in molecular sieve. ${ }^{[14]}$ Interestingly, a related high resolution TEM tomography study proved that the produced branches were slightly thicker (ca. $3 \mathrm{~nm}$ ) than the typical pore diameter $(\mathrm{ca} .2 \mathrm{~nm}$ for MCM-41). ${ }^{[5]}$ 
WILEY-VCH

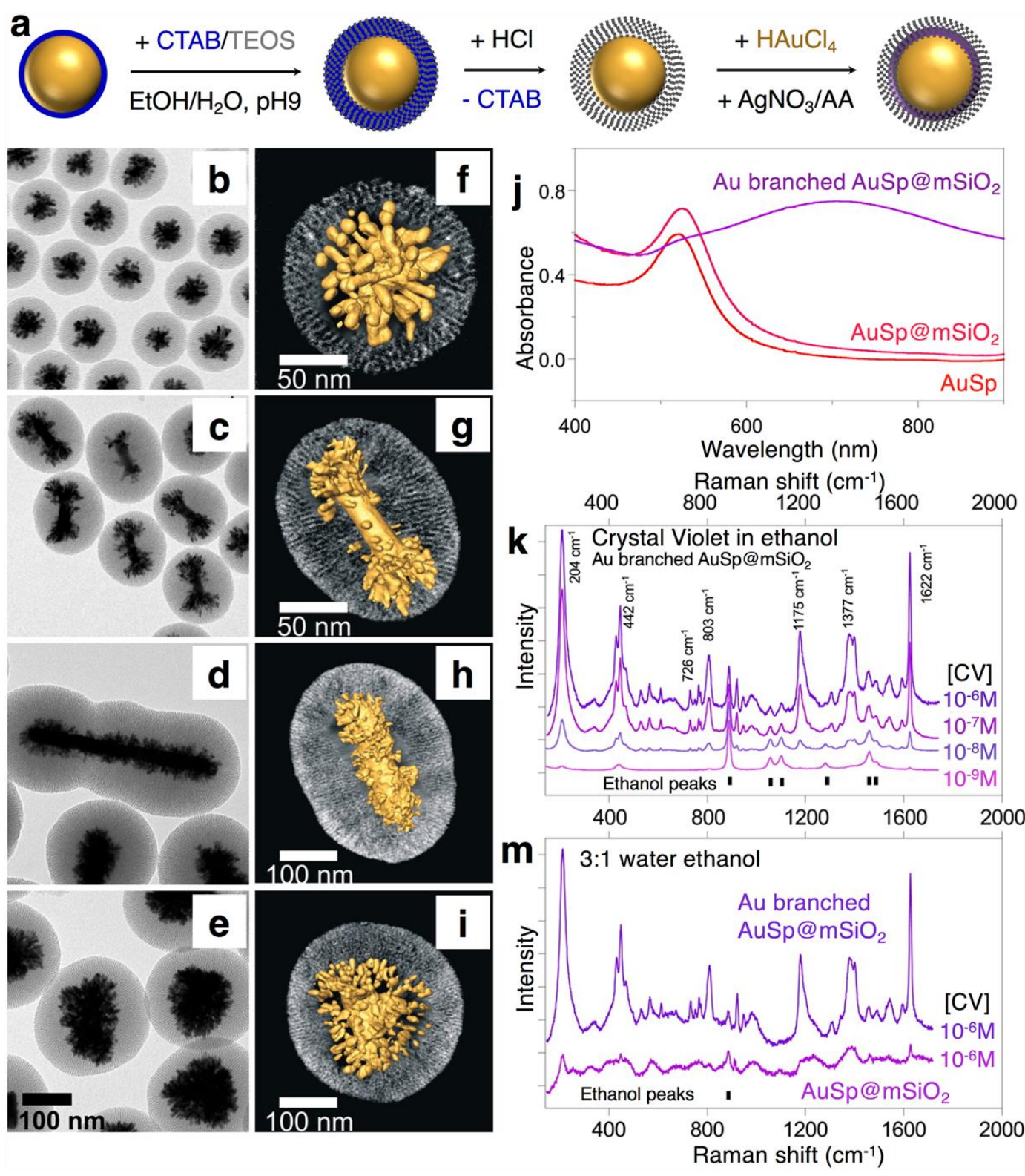

Figure 6. Template-assisted synthesis of branched gold nanoparticles encapsulated in mesoporous silica shells, for SERS applications. a) Scheme of the fabrication of $\mathrm{Au} / \mathrm{mSiO}_{2}$ core-shell particles in the presence of CTAB (blue), scaffold removal by washing with $\mathrm{HCl}$ in $\mathrm{EtOH}$, and subsequent growth of gold tips (purple layer) in water through the open pores in the presence of silver ions. b-e) TEM micrographs (from top to bottom) of gold spheres, singlecrystal rods, pentatwinned rods, and triangles after encapsulation and branching. f-i) Corresponding 3D reconstructions obtained by electron tomography. j) UV/vis extinction spectra of $15 \mathrm{~nm} \mathrm{Au}$ spheres before (AuSp) and after $\left(\mathrm{AuSp} @ \mathrm{mSiO}_{2}\right)$ encapsulation with silica, as well as of the resulting branched $\mathrm{Au} / \mathrm{mSiO}_{2}$ particles, showing negligible aggregation during silica growth (slight red-shift attributable to refractive index increase) and a pronounced LSPR red-shift upon tip growth. k) SERS spectra of crystal violet adsorbed on branched $\mathrm{Au} / \mathrm{mSiO}{ }_{2}$ nanospheres, dispersed in EtOH. m) Comparison of the SERS intensity of pristine and branched $\mathrm{Au} / \mathrm{mSiO}_{2}$ nanospheres, showing a significant signal enhancement due to the branches. Adapted under the terms of the ACS AuthorChoice license. ${ }^{[14]}$ Copyright 2015, American Chemical Society. Permission requests for the material excerpted should be directed to the ACS. (http://pubs.acs.org/doi/abs/10.1021\%2Facsnano.5b04744) 


\section{WILEY-VCH}

Besides functioning as a permanent hard template to direct oriented metal growth while providing stability, silica can also be used as a sacrificial layer. In the proximity of a metal/metal oxide interface, non-sintered silica displays a low crosslinking density and, as a result, quickly dissolves in (basic) aqueous media, which can lead to a hollowing out etching process, eventually resulting in yolk-shell or rattle type particles. ${ }^{[118,119]}$ As shown in Figure 7, this effect can be alleviated by thermal annealing of the reaction mixture, but it can also be used to produce more complex nanostructures resembling matryoshka dolls. ${ }^{[88]}$ Furthermore, after coating with a porous shell of another, more hydrolysis resistant material, the silica interlayer can be easily removed, resulting in well-defined nanoreactors. This approach has been often used with metal oxides such as $\mathrm{TiO}_{2}, \mathrm{ZrO}_{2}$, or $\mathrm{CeO}_{2}$ to produce nanocatalysts. ${ }^{[59]}$ An interesting method for the synthesis of easily dispersible yolk-shell structures was presented by Dillon et al., in the form of a $\mathrm{Au} / \mathrm{SiO}_{2} / \mathrm{TiO}_{2} / \mathrm{SiO}_{2}$ ternary shell configuration, which facilitated redispersion of the particles after thermal annealing by simply dissolving both silica shells in dilute $\mathrm{NaOH}$ solution at $90{ }^{\circ} \mathrm{C}^{[120]}$

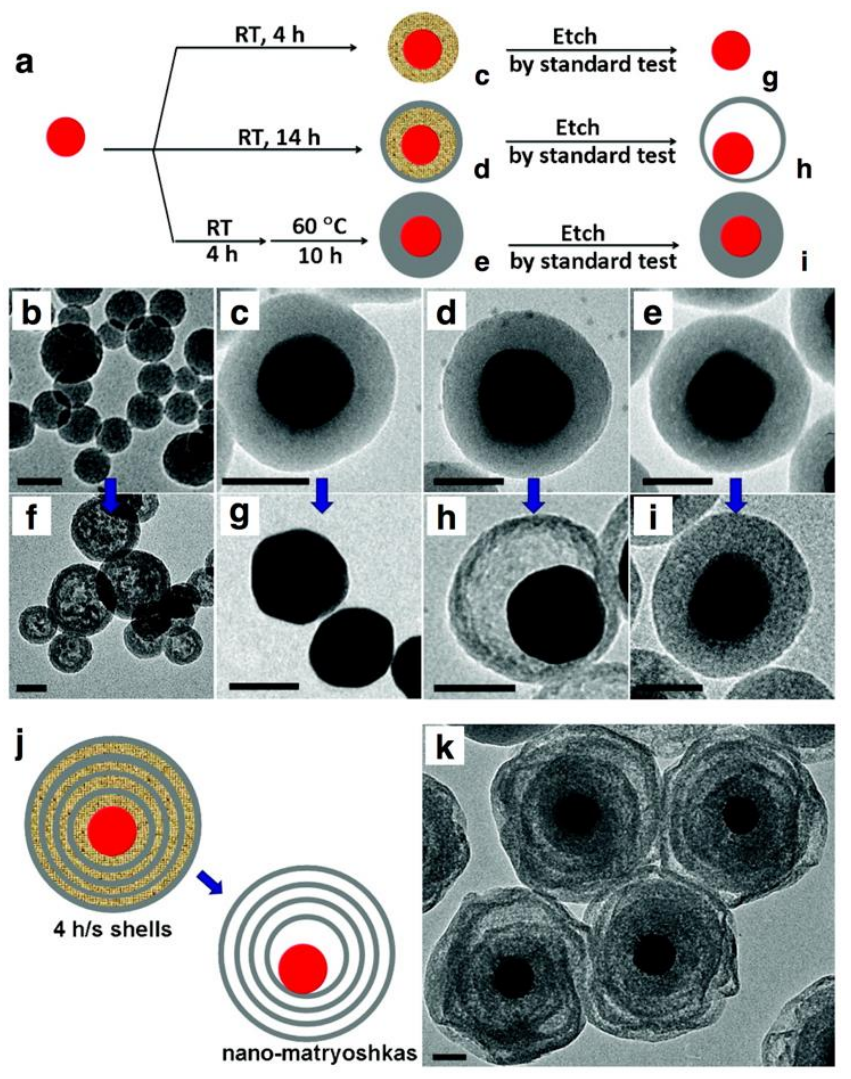




\section{WILEY-VCH}

Figure 7. Fabrication of yolk-shell particles and nanomatryoshkas using the chemical inhomogeneity of silica shells prepared by conventional Stöber synthesis. a) Scheme of silica growth and subsequent etching: whereas silica growth for short periods at room temperature yielded a weakly crosslinked network, longer reaction times and higher temperature resulted in hardening of the shell from the outside toward the inside. b-e) TEM images of bare silica particles cured at room temperature for $14 \mathrm{~h}$ and of $\mathrm{Au} / \mathrm{SiO}_{2}$ core-shell particles hardened by curing under the conditions indicated in (a). f-i) TEM images of the corresponding particles after hydrothermal etching for $30 \mathrm{~min}$ at $90{ }^{\circ} \mathrm{C}$. j,k) Synthesis of nanomatryoshkas via multistep overgrowth with inhomogeneous silica layers followed by etching. Adapted with permission. ${ }^{[88]}$ Copyright 2011, American Chemical Society.

\section{Applications of Plasmonic Metal/SiO 2 Hybrid Particles}

Progress in the large-scale synthesis of well-defined hybrid nanoparticles has opened exciting avenues for the utilization of plasmonics in a wide range of applications. We discuss in this section a selection of the most successful implementations of noble metal/silica nanocomposites, namely in sensing, theranostics, nanoparticle catalysis, and plasmonic enhancement of thin film solar cells. It will be shown that the role of the silica shells, though often modest, is essential toward the development of these applications. As it was introduced in section 2, and summarized in Figure 1, silica coatings allow us to exploit the remarkable properties of noble metal nanoparticles by conferring them with colloidal, chemical and thermal stability, allowing controlled coupling for plasmon-enhanced optical applications, or providing electrical insulation in solar cells. Moreover, in the particular case of mesoporous silica, the coating may endow the particles with further functionalities, acting as a template to change the geometry of the cores, allowing selective sieving of analytes diffusing toward the core, or providing a cargo space for drug delivery applications.

\subsection{Nanocomposites for Sensing}

\subsubsection{Plasmonic sensors}

Highly sensitive and, ideally, portable sensing devices offering fast and cost effective ex situ analysis are in high demand and their fields of application include, among others: monitoring of air and water quality by detection of hazardous chemicals, drugs and explosives tests in 


\section{WILEY-VCH}

forensics, pregnancy tests, glucose level measurements, and tracing of biological markers in urine or blood for both early diagnosis and long-term disease monitoring. In all of these examples, the available amount of sample is usually small. For some applications, the analysis is just intended to provide a yes/no answer regarding the presence of an analyte, whereas others require quantitative determinations. A straightforward strategy in sensing applications comprises focusing on the detection of analytes that modify intrinsic, well-known features of the selected probe. Noble metal nanoparticles owe their successful implementation in a plethora of sensing applications mainly to two characteristics: sensitivity of the optical LSPR frequency to changes in the dielectric environment around the particle, and plasmon coupling occurring when the particles come close to each other, which gives rise to distinct color shifts as well as strongly enhanced electromagnetic fields. As a consequence, many sensing systems have been developed for the detection of analytes that can potentially alter the size, shape, the state of aggregation of the colloidal probes, or the dielectric function of the medium. ${ }^{[121,122]}$

\subsubsection{LSPR Sensing}

In many fundamental works on plasmonic hybrid materials, silica coatings have been used to tune the refractive index around nanoparticles to study its influence on the position of the LSPR band. ${ }^{[36,123]}$ Besides directly inducing LSPR shifts, thin silica layers have also been shown to change the sensitivity of the peak position toward the adsorption of molecules and of proteins in particular. In the case of mesoporous coatings, the resulting LSPR shift is associated to the refractive index corresponding to an effective medium made of silica and the solvent (or air). This property has been used to adjust the sensitivity range, as demonstrated for optical fiber sensors in which the LSPR of nanostructured gold surfaces served to monitor the refractive index variation of a medium contained inside channels coated with mesoporous silica films of varying thickness. ${ }^{[124]}$ Due to the small sensing volumes contributing to LSPR shifts, precise control over device geometry is of key importance and consequently, methods to deposit thin silica layers onto plasmonic substrates have been developed. ${ }^{[125]}$ Since silica also features 


\section{WILEY-VCH}

compatibility with biological and biomimetic systems, such composite substrates consisting of gold nanodisks covered with a $5 \mathrm{~nm}$ silica layer could be further equipped with a lipid bilayer to monitor the binding of a cholera toxin. An interesting concept for an LSPR-based sensor that permits gas detection was introduced by Tittl et al. ${ }^{[126]}$ Thereby, hydrogen adsorption was followed through the LSPR red-shift of $\mathrm{Au} / \mathrm{SiO}_{2}$ particles coated with a thin Pd film. ${ }^{[126]}$ The $10 \mathrm{~nm}$ silica shell functioned as a thin spacer and intriguingly, in the reverse geometry where $\mathrm{Pd}$ acted as a plasmonic mirror underneath the $\mathrm{Au} / \mathrm{SiO}_{2}$ particles hydrogen adsorption caused a blue-shift of the extinction maximum. As illustrated in Figure 8a-g, formation of PdH changed the metallic character of the Pd substrate modifying the coupling strength and resonance frequency of the hybridization modes of the mirror surface and the shell-isolated nanoparticles (SHINs).
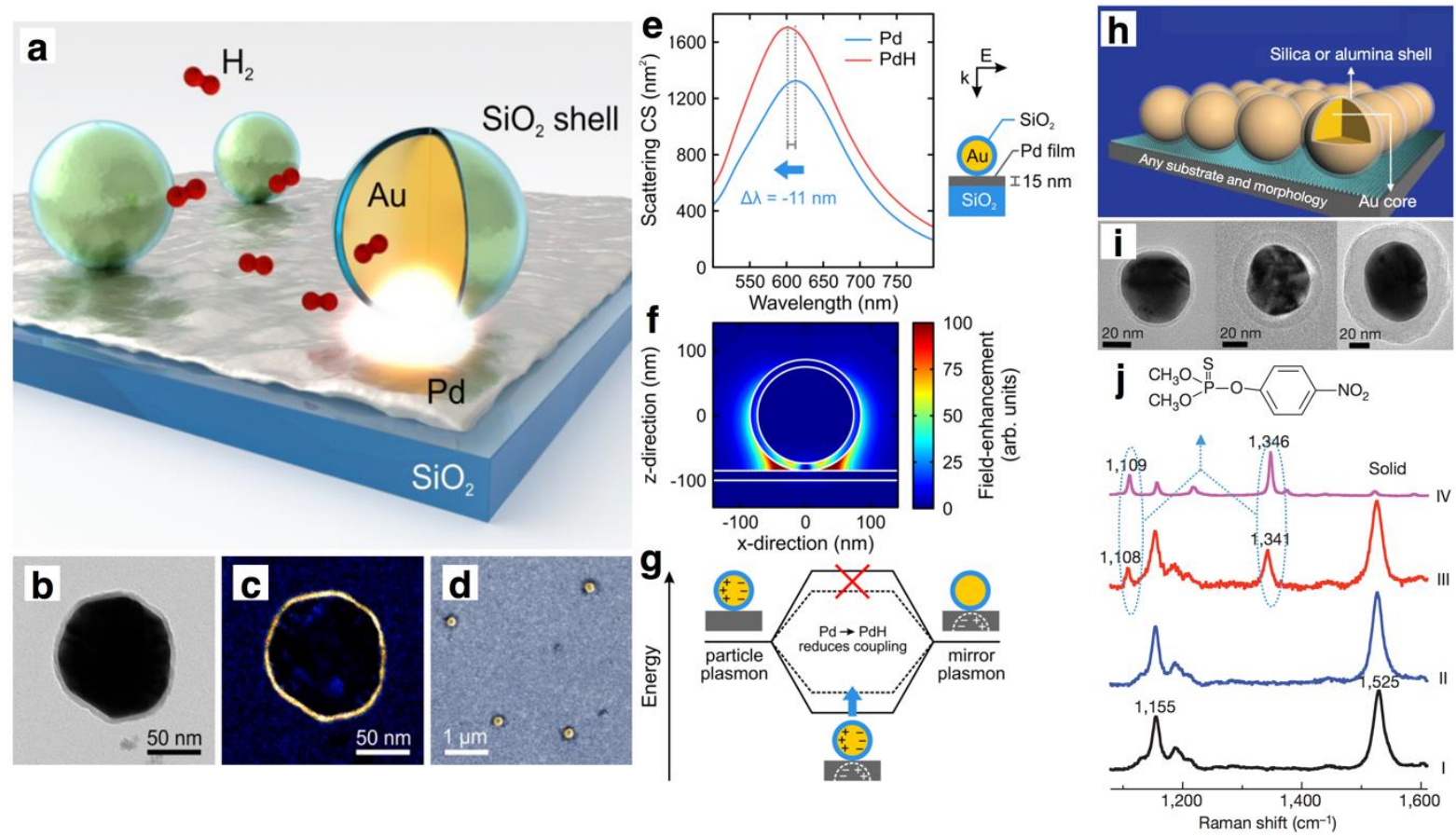

Figure 8. Reaction monitoring by sensing with $\mathrm{Au} / \mathrm{SiO}_{2}$ NPs. a) Illustration of a SHIN-based substrate for probing hydrogen binding to Pd surfaces. b) TEM micrograph of a typical $\mathrm{Au} / \mathrm{SiO}_{2}$ probe with a core diameter of $150 \mathrm{~nm}$ and a $10 \mathrm{~nm}$ shell. c) Energy-filtered TEM image taken at the Si L-edge. d) SEM micrograph of a Pd surface after SHIN deposition. e) Modelled spectra of a core-shell particle on Pd before (blue curve) and after (red curve) hydrogen binding demonstrating a $11 \mathrm{~nm}$ blue-shift due to $\mathrm{PdH}$ formation. f) Simulated distribution of the electric near-field under resonant excitation. g) Hybridization model showing a dark anti-bonding and bright bonding mode. Diminishing the metallic character of the Pd mirror weakens the coupling strength leading to a blue-shift of the observable low energy hybrid mode. Adapted with 


\section{WILEY-VCH}

permission. ${ }^{[126]}$ Copyright 2013, American Chemical Society. h) Scheme of SERS sensing using SHINERS. i) TEM micrographs $\mathrm{Au} / \mathrm{SiO}_{2} \mathrm{NPs}$ with varying shell thickness. j) Detection of pesticide residues via Raman and SHINERS: I) Raman spectrum recorded on a clean orange, II) Raman on a contaminated fruit, III) SHINERS on a contaminated fruit, IV) Raman of solid parathion. With SHINERS a clear signal for the characteristic peak around $1346 \mathrm{~cm}^{-1}$ was detected, whereas the conventional Raman measurement remained silent in that spectral region. Adapted with permission. ${ }^{[45]}$ Copyright 2010, Macmillan Publishers Ltd.

\subsubsection{Surface Enhanced Raman Scattering}

The most prominent plasmonic sensing applications are most likely those based on near-field enhancement. When illuminated by light with wavelength at the resonance frequencies of plasmonic nanostructures, these act as antennas that efficiently confine incident light into a small volume close to their surface, often achieving an increase in the electric field of over a thousand-fold. ${ }^{[127]}$ Surface enhanced Raman scattering spectroscopy is the prime example of optical sensing techniques that exploit this characteristic behavior to improve sensitivity. ${ }^{[128]}$ Raman spectroscopy is a very precise technique for the unequivocal identification of molecules, through their unique vibrational fingerprint. However, most Raman-active substances yield very weak signals due to small molecular scattering cross-sections, ranging from $10^{-27} \mathrm{~cm}^{2} \mathrm{sr}^{-1}$ for certain dyes, down to $10^{-30} \mathrm{~cm}^{2} \mathrm{sr}^{-1}$ for non-resonant molecules. ${ }^{[127]}$ The Raman crosssection scales with the fourth power of the electric field of the exciting light, meaning that analytes within the near-field of resonantly excited plasmonic particles can witness Raman scattering enhancements up to 12 orders of magnitude, thereby allowing in certain cases even single molecule detection. ${ }^{[129,130]}$

Near-field enhancement strength depends mainly on the size, shape, and the aggregation state of the employed nanostructures. The highest enhancements are achieved at the so called hotspots, which are generally located at sharp-edged features due to the lightning rod effect or within strongly coupled particle clusters. ${ }^{[26,131]}$ Consequently, colloidal stability and reproducible interparticle spacing are essential for quantitative SERS measurements. In this regard, silica coatings may play an essential role. In relatively few cases a dense silica layer has 


\section{WILEY-VCH}

been chosen for the protection of plasmonic particles, an example being the selective detection of metal cations that can permeate the shell. ${ }^{[78]}$ By using this approach, the presence of ions was monitored through the shift of a SERS peak of sodium 2-mercaptoethanesulfonate, surrounding the cores of $\mathrm{Ag} / \mathrm{SiO}_{2}$ particles. Another application of hybrid particles with closed shells in SERS is shell-isolated nanoparticle enhanced Raman spectroscopy, or SHINERS, as introduced by Tian's group. ${ }^{[45,132]}$ Thereby, noble metal nanoparticles with silica coatings of 2-4 nm were spread over analyte-bearing substrates, to act as plasmonic tips that enhance the local optical fields up $142 \times$ without electronically or chemically interacting with the probed molecules. Moreover, the silica spacer in this system provides protection against uncontrolled nanoparticle aggregation, which would result in irregular coupling and in turn irreproducible SERS enhancement. ${ }^{[46]}$ Apart from reaction monitoring, SHINERS has been employed, for instance, in pesticide detection, as shown in Figure $\mathbf{8 h - j}$, or for breast cancer type identification. ${ }^{[45,133]}$ Mesoporous silica is however usually preferred, as it provides protection to the particles while allowing the diffusion of analytes toward the plasmonic surface. As shown in Figure 6, mesoporous silica coatings with radially oriented channels can be employed to template the growth of Au tips from encapsulated Au cores, achieving high colloidal stability in the absence of surfactants or polymeric ligands. ${ }^{[14]}$ The analyte, in this case crystal violet (CV), could therefore diffuse through the open pores and directly access the hot-spots at the tips of the branches, leading to strong Raman signal enhancement as compared to the unmodified, spherical counterparts. To improve the detection limit of molecules with a small Raman scattering cross-section, molecular recognition leading to a preferential binding of analyte to the hot spots can be employed. For instance, an increase of up to two orders of magnitude compared to previous methods in the detection of enrofloxacin by SERS was achieved by coating spiked $\mathrm{Au} / \mathrm{mSiO}_{2}$ particles with a molecularly imprinted polymer with specific affinity toward this antibiotic. ${ }^{[134]}$ This molecule was therefore preferentially placed at the hot-spots and was consequently detected by SERS. Another common strategy to enhance the SERS signal, 


\section{WILEY-VCH}

and thereby improve the sensitivity of molecular detection, is related to increasing the density and enhancement strength of the hot-spots. ${ }^{[135]}$ An interesting example of such an optimization of plasmonic hybrids has been reported by Panikkanvalappil and El-Sayed, who used a thin silica shell as a spacer between a rough Au substrate (Au mesoflowers) and a dense film of small citrate-capped gold particles. ${ }^{[136]}$ In this case, the role of the silica layer was two-fold; protecting the spiked underlying surface from reshaping and providing amino-functionalized anchoring points for the adsorption of gold nanoparticles. The high density of plasmonic hotspots thus obtained enabled the detection of complex molecules such as a prostate-specific antigen (PSA) from serum. The accessible surface area for analyte adsorption, and therefore the number of hot-spots, can be further increased by using multilayered substrates. This has been demonstrated via the hierarchical organization of gold nanorods into lamellar supercrystals by templated self-assembly, using predefined hole patterns as templates. The obtained supercrystals were subsequently incubated with a silica sol-gel precursor solution, resulting in the formation of a mesoporous film, both surrounding the outer faces and intercalating between neighboring nanorod monolayers. ${ }^{[137]}$ The silica film not only protected the supercrystals from detaching but also led to increased overall enhancements of the SERS performance and could further be used as a molecular sieve allowing only small molecules to diffuse into the hot-spots. As an end application, the biocompatible mesoporous silica surface allowed culturing bacterial colonies and investigating in situ bacterial communication by SERS. More specifically, Bodelón et al. monitored the expression of pyocyanine, a molecule involved in the quorum sensing cycle of Pseudomonas aeruginosa, down to sub-nanomolar concentrations, i.e. from the onset of colony interactions up to the stage of biofilm formation, as displayed in Figure 9. ${ }^{[80]}$

Although, as we have seen, silica coating is typically used to preserve the plasmonic properties of the nanostructured metals, it has been demonstrated that it can also provide additional functionalities. An example from Tang's group is related to coating of gold nanorods with 


\section{WILEY-VCH}

mesoporous silica synthesized in the presence of chiral surfactants, resulting in enhanced optical activity. ${ }^{[138]}$ The circular dichroism signal of the composite colloidal system was shown to depend on both the surfactant chosen for the synthesis and the aspect ratio of the gold nanorods. Intriguingly, this system was also used for SERS-based enantiomer recognition, as the spectral details were shown to depend on the chirality of the molecules loaded inside the mesopores.
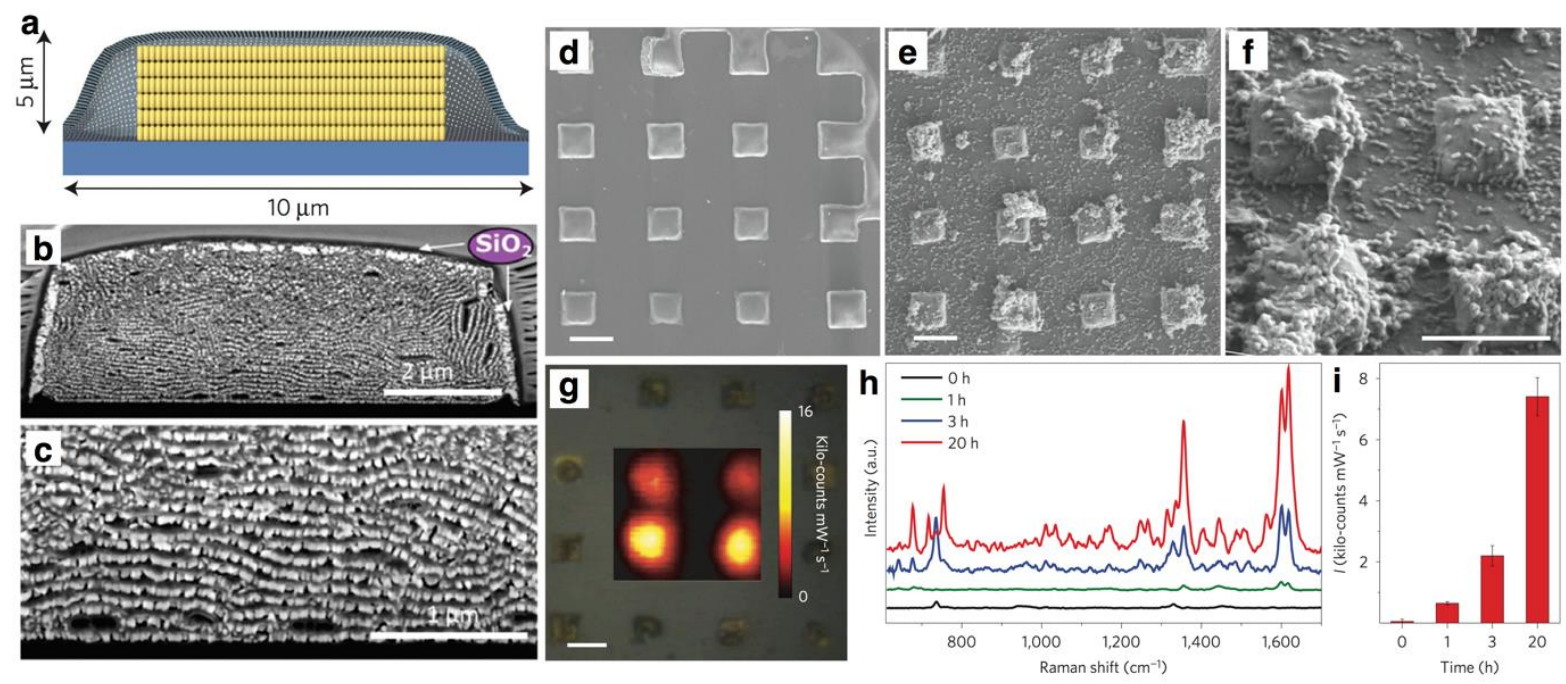

Figure 9. AuNR/mSiO 2 arrays for SERS detection of small molecules in complex, biological media. a) Schematic depiction of an array composed of stacked NR lamellae protected by a mesoporous silica coating. b,c) Cross-sectional SEM-FIB micrographs of a cuboid-shaped supercrystal. d) SEM image of a pristine AuNR/mSiO 2 array. e,f) The same substrate after incubation with Pseudomonas aeruginosa. g) Reflected light micrograph with superimposed SERS map, representing the local pyocyanine SERS intensity at $1600 \mathrm{~cm}^{-1}$. h) SERS spectra of pyocyanin obtained on $\mathrm{AuNR} / \mathrm{mSiO}_{2}$ arrays with excitation at $785 \mathrm{~nm}$, after different bacterial proliferation times. i) SERS signal intensities after 0-20 hours of incubation with bacteria. a,di) Adapted with permission. ${ }^{[80]}$ Copyright 2016, Macmillan Publishers Ltd. b,c) Adapted under the terms of the CC BY 3.0 license. ${ }^{[137]}$ Copyright 2016, published by The Royal Society of Chemistry. (http://dx.doi.org/10.1039/C6NR00712K)

\subsubsection{Surface Enhanced Fluorescence}

Plasmonic near-field enhancement has been shown to enhance the light emission by fluorophores located in close vicinity of metallic nanostructures, which is known as surface enhanced fluorescence (SEF) and illustrated in Figure 10 for gold nanoshells. ${ }^{[139,140]}$ However, in contrast to SERS, direct contact between the plasmonic particle surface and the analyte results in luminescence quenching by non-radiative processes, whereas at distances of several 


\section{WILEY-VCH}

to tens of nanometers luminescence is enhanced. ${ }^{[141]}$ Therefore, a thin metal oxide layer is commonly used as a stable insulating spacer, whose thickness can be precisely tuned to optimize SEF. ${ }^{[42]}$ Although the optimal distance depends on the type of nanoparticle and the overlap between its plasmon resonance band and the emission band of the fluorophore, typical values range from 5 to $20 \mathrm{~nm}$ often resulting in an enhancement factor of more than $10 .^{[140-143]}$ In SEF-based sensing applications, functionalization of the silica spacer is often exploited to achieve selective recognition for certain analytes. For instance, DNA tagged with a fluorophore displaying specific recognition has been used to detect traces of mercury. ${ }^{[144]}$ Niu et al. developed a switch-on technique for the detection of pyrophosphate in living cells and assays. ${ }^{[145]}$ Their system comprised silica-coated gold nanoparticles with a NIR luminescent dye attached to the shell, whose luminescence was quenched by the binding of $\mathrm{Cu}^{2+}$ ions and recovered in the presence of pyrophosphate, which captured $\mathrm{Cu}^{2+}$ due to its higher affinity. Interestingly, plasmonic systems allowing for simultaneous SERS and SEF enhancements have been devised. In a recent example, Ag nanocubes separated from an alumina-coated aluminum foil by a thin silica spacer were employed to detect the concentration of creatinine and flavin adenine dinucleotide coenzyme in urine through both techniques. ${ }^{[146]}$

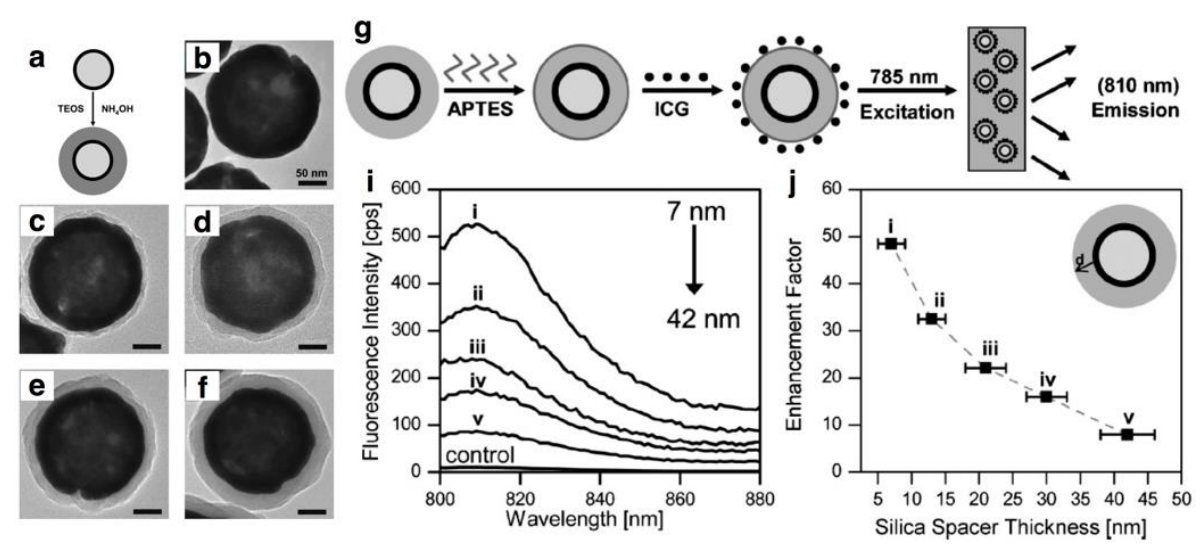

Figure 10. Spacer controlled fluorescence enhancement by $\mathrm{Au}$ nanoshells (AuNSHs). a) Scheme of silica coating on AuNSHs b-f) TEM images of AuNSHs coated with 7, 13, 21, 30, and $42 \mathrm{~nm}$ silica layers, respectively (all scale bars: $50 \mathrm{~nm}$ ). g) Illustration of fluorophore conjugation and optical characterization. i) Fluorescence spectra of Indocyanine Green (ICG) conjugated at the corresponding distances from the Au-NS surface (i-v: 7-42 nm) and spectrum 


\section{WILEY-VCH}

of silica NPs used as a control. j) Fluorescence enhancement factor as a function of silica shell thickness showing approximately $80 \%$ signal decay as the spacer thickness increases from 7 to $42 \mathrm{~nm}$. Adapted with permission. ${ }^{[140]}$ Copyright 2008, Wiley-VCH Verlag GmbH \& Co. KGaA, Weinheim.

\subsection{Theranostics}

\subsubsection{Nanomedicine}

With specifically functionalized nanoparticles opening up avenues for personalized treatments, it has become evident that medicine is one of the most promising application fields of nanotechnology. ${ }^{[147]}$ The most widespread subject of research in nanomedicine has been cancer diagnosis and treatment, which is owed not only to the high prevalence and mortality of the disease, but also to the fact that many types of cancer need to be addressed in different ways and demand highly specific medication. In contrast to conventional systemic chemotherapy, i.e., the introduction of cytotoxins addressing fast-dividing cells through the bloodstream, targeted therapies based on nanoparticles aim at the accumulation of anti-cancer agents inside the tumor tissue, potentially enabling the use of more aggressive treatments while minimizing side effects. This objective can be accomplished via local injection, active guiding of responsive drug carriers under external fields, or by passive targeting, whereas the most advanced targeting concepts are based on surface functionalization with specific antibodies or biorecognition entities for glycoproteins that are typically overexpressed by tumor cells. ${ }^{[147,148]}$ Great effort is being devoted to confer multifunctional medication systems based on nanoparticles fitted to target a specific type of cancer, while at the same time allowing to image the tumor, determine its characteristics, and finally commence treatment. This multipurpose set of features is currently identified by the term theranostics. Most of the diagnostic methods used therein are derived from the sensing techniques discussed above. In this context plasmonic nanoparticles can be employed for mapping of SERS, SEF or other signals, and silica shells are widely applied because their straightforward chemistry provides biocompatibilization and means for the attachment of targeting moieties, as well as the transient incorporation of drug molecules. 


\section{WILEY-VCH}

\subsubsection{Nanocomposite-Assisted Imaging Techniques}

A wide variety of plasmonic nanoparticles have been tailored for use as imaging tags for optical cell culture monitoring, or even for in vivo applications. Biocompatibility is of paramount importance here and deep tissue imaging requires LSPR bands being localized in the near infrared (NIR) region, so as to minimize absorption and scattering by biological tissue constituents, in turn increasing penetration depth. ${ }^{[149]}$ Most of the composite particles described so far are intrinsically biocompatible because of the presence of silica shells. Gold nanorods and hollow gold nanoshells have been used most often for biological applications, as their longitudinal LSPR resonance can be routinely tailored during synthesis to match the desired biological window. ${ }^{[150]}$ Additionally, nanocubes, nanostars, dimers, yolk-shell particles or nanobipyramids have also been employed..$^{[75,99,145,151,152]}$ For example, Niu et al. used silicacoated AuNRs and AuNBPs in combination with NIR dyes to image cancer cells through SEF. ${ }^{[145]}$ The authors employed a switch-on technique that allowed them to observe luminescence exclusively in the presence of pyrophosphate, a molecule highly expressed in tumor and other rapidly dividing cells. Cell imaging via SERS has also become an established method and can be conducted by combining plasmonic particles, Raman reporters and targeting moieties for specific cells or membrane types. ${ }^{[99,151,152]}$ As displayed in Figure 4, excitation and emission bands of Raman dyes are narrow, and consequently multiplexing via excitation of multiple dyes with different laser lines can be accomplished more easily than with the equivalent fluorescence-based imaging probes. ${ }^{[28,153]}$ In fact, in vivo imaging of tumors, in which SERS-encoded nanoparticles accumulate preferentially, has been demonstrated. ${ }^{[154]}$ Nowadays, increasingly complex hybrid nanoparticle architectures incorporating multiple functionalities are being developed, which enable simultaneous multimodal imaging by complementary techniques. Wang et al. implemented a multilayered silica coating process to incorporate a magnetic core, silver-coated AuNRs, Raman tags and quantum dots within a single colloidal object, which was further functionalized with antibodies to target and image up 


\section{WILEY-VCH}

to five different cell lines, both by SERS and luminescence. Subsequent recovery of the cells was possible by applying a magnetic field. ${ }^{[155]}$ Apart from facilitating optical imaging, gold nanoparticles can also be used for imaging by other methods, among which photoacoustic tomography (PAT) has acquired a high relevance. In PAT, the geometry of an object is obtained by analyzing the acoustic signal generated by the thermoelastic expansion of a contrast agent upon excitation with a pulsed laser. It has been shown that silica coating of AuNRs not only significantly improves their uptake into cells, ${ }^{[63]}$ but further amplifies their photoacoustic signal by up to three times. ${ }^{[156]}$ Sharma et al. selected silica particles decorated with gold particles and proposed that their PAT imaging capability would be improved by increasing the number of dielectric-metal interfaces. ${ }^{[157]}$ Further doping with gadolinium has also been shown to allow magnetic resonance imaging (MRI) as an additional imaging modality. Due to the high electron density of gold, AuNRs can also replace iodine as a contrast agent for X-ray Computed Tomography (CT) imaging. ${ }^{[65]}$ An example of an advanced noble metal/silica system featuring three complementary imaging modalities is displayed in Figure 11. ${ }^{[158]}$ The hybrid multifunctional particles used in this study, which the authors called quantum rattles $(\mathrm{QR})$, were composed of a mesoporous silica shell containing $\mathrm{Au}$ nanoparticles and Au quantum dots, which are paramagnetic and photoluminescent, trapped in its pores. These nanostructures were applied for NIR fluorescence, photoacoustic and magnetic resonance imaging, but also offered therapeutic functionalities. 

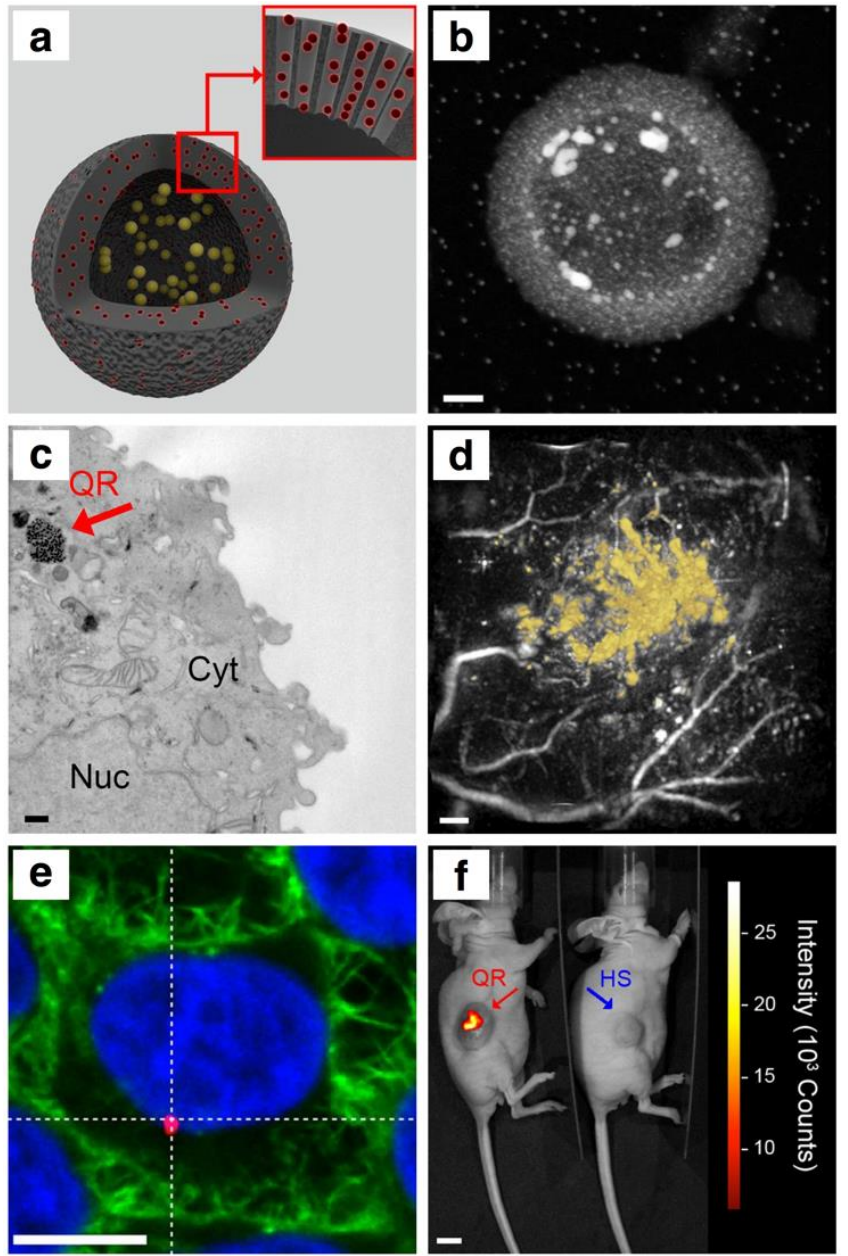

Figure 11. a) Illustration of quantum rattles (QRs) for multimodal imaging and phototherapy, comprising a hollow mesoporous silica capsule carrying $2 \mathrm{~nm}$ sized Au quantum dots (red) in the pores and larger gold nanoparticles inside the cavity (yellow). b) HAADF-STEM image of a QR showing the encapsulated AuNPs as bright white dots (scale bar: $20 \mathrm{~nm}$ ). c) TEM micrograph of an ultrathin section of an incubated HeLa cell proving the internalization of the QRs (scale bar: $500 \mathrm{~nm}$ ). d) 3D photoacoustic image of a tumor, acquired at $670 \mathrm{~nm}$ after injection of QRs. e) Laser confocal scanning microscopy image of a HeLa cell showing the accumulation of QRs in the perinuclear region through their own fluorescence (red). f) NIR fluorescence images showing the injection site in tumors for QRs (red) and non-fluorescent control (HS, blue) sample (scale bar: $10 \mathrm{~mm}$ ). Adapted with permission. ${ }^{[158]}$ Copyright 2015, published by National Academy of Sciences. (http://dx.doi.org/10.1073/pnas.1419622112)

\subsubsection{Photothermal Therapy}

Plasmonic nanoparticles of lossy materials such as gold display strong optical absorption at their LSPRs, usually leading to significant heat dissipation into the particle surrounding upon decoherence of the excited plasmon. This property is widely used for photothermal therapy. ${ }^{[159]}$ Similar to bioimaging, gold nanorods and nanoshells (AuNSHs) are obvious choices for core particles due to their optical properties, ${ }^{[160]}$ while gold nanocages and nanostars have also been 


\section{WILEY-VCH}

explored to a lesser extent. ${ }^{[72]}$ In hyperthermia treatments, the basic therapy consists of inducing a temperature increase that provokes cell apoptosis (temperatures above $42{ }^{\circ} \mathrm{C}$ ) or necrosis (above $80^{\circ} \mathrm{C}$ ) by inducing the rupture of the cell membrane, preventing DNA and RNA replication and inducing protein denaturation. ${ }^{[148]}$ In order to control heat dissipation while avoiding thermal reshaping of the metal particles and the corresponding LSPR shifts, ${ }^{[161]}$ silica coatings have been commonly applied. Chen et al. improved the capabilities of their theranostic nanocomposites by an unusual approach using upconverting particles based on rare earth ions. Excitation with NIR frequencies thus leads to the emission of green and red light, while the thickness of the silica spacer could be optimized to match the emitted light to the LSPR band of the gold nanoparticles, favoring energy transfer and conversion into heat for hyperthermia. ${ }^{[162]}$ As demonstrated by Rohani et al., by combining silica-coated upconverting nanocrystals (UPNCs) with AuNRs, not only luminescence enhancements and optical heating capabilities can thus be realized, but also temperature monitoring via analysis of the UCNP emission properties. ${ }^{[163]}$

\subsubsection{Drug Delivery}

Mesoporous silica shells can add a further therapeutic utility to the hybrid particles, by enabling the incorporation of drug molecules. ${ }^{[164,165]}$ Thereby, plasmonic heating may serve a double purpose, both inducing hyperthermia and triggering the release of encapsulated drugs. ${ }^{[70,71]}$ These two roles may act synergistically for cancer treatment, exploiting the increased cell membrane permeability at high temperature, which leads to improved diffusion of drug molecules into cells. ${ }^{[75]}$ For this purpose, Zhang et al. designed AuNR/mSiO 2 nanocomposites loaded with doxorubicin, a common chemotherapy agent, such that low intensity NIR irradiation triggered drug release, whereas excitation at higher power caused hyperthermia (Figure 12). ${ }^{[70]}$ Enhanced drug loading capacity was demonstrated by using hollow metal nanoparticles such as Au nanocages. ${ }^{[72]}$ A similar approach used coated silica particles with small magnetic particles and a gold layer, so that subsequent dissolution of the silica cores 


\section{WILEY-VCH}

produced nanoparticles that could be loaded with a cargo and used for both magnetic resonance imaging and hyperthermia. ${ }^{[166]}$
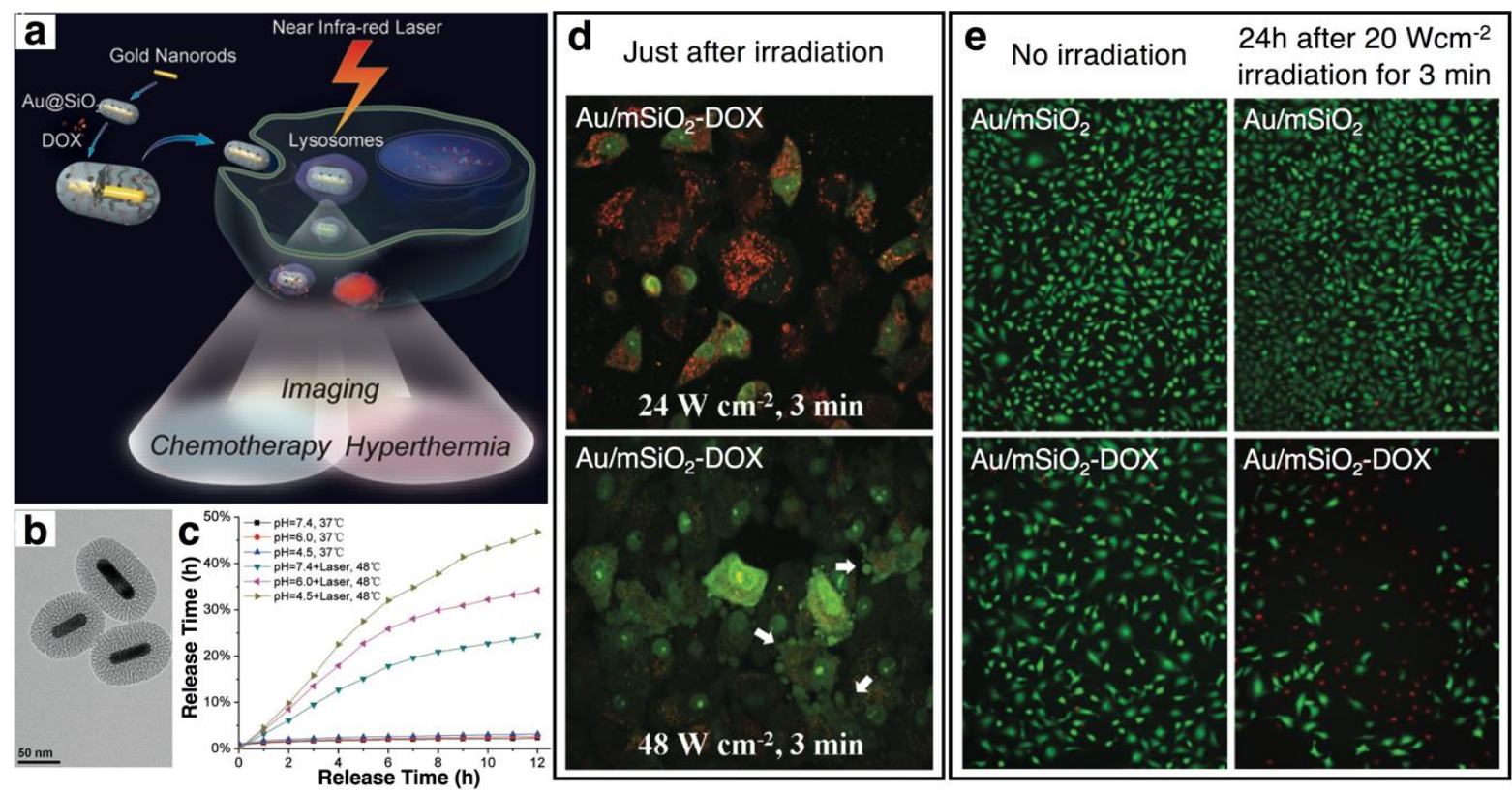

Figure 12. Mesoporous silica-coated AuNRs loaded with doxorubicin (DOX) for multimodal therapy: chemotherapy and hyperthermia. a) Schematic representation of the preparation of $\mathrm{Au} / \mathrm{mSiO}_{2}-\mathrm{DOX}$ particles and their application in cells. After internalization, the particles are excited via irradiation with an NIR laser, which allows optical imaging as well as inducing cell death by heating and drug release. b) Representative TEM image of the particles. c) Time dependent DOX release at different $\mathrm{pH}$ values and temperatures, showing negligible release at $37{ }^{\circ} \mathrm{C}$ and around 30-45\% release upon heating to $48{ }^{\circ} \mathrm{C}$. d) Effect of NIR laser (790 nm) on heating the lysosomal membrane of A549 cells incubated with nanoparticles: irradiation with a power density of $24 \mathrm{~W} \mathrm{~cm}^{-2}$ led to temperature-induced DOX release, but no lysosomal damage was observed. At $48 \mathrm{~W} \mathrm{~cm}^{-2}$ rupture of the lysosomal membrane due to hyperthermia was evident. e) Effect of therapeutic treatment with (DOX-)particles after incubation for $12 \mathrm{~h}$ $\left(\mathrm{Au} / \mathrm{mSiO}_{2}\right.$ was used as control): the most efficient killing after $24 \mathrm{~h}$ was observed for DOXparticle treated cells upon irradiation for $3 \mathrm{~min}$ at $20 \mathrm{~W} \mathrm{~cm} \mathrm{~cm}^{-2}$ (living cells: green, dead cells: red). Adapted with permission. ${ }^{[70]}$ Copyright 2012, Wiley VCH Verlag GmbH \& Co. KGaA, Weinheim.

\subsubsection{Targeting Strategies}

Selective accumulation inside tumors is of key importance toward the efficient application of nanoparticles in cancer therapy, and therefore significant effort has been devoted to the development of administration strategies. Allowing for both diagnosis and treatment, systemic administration through the bloodstream is most often desirable. Due to the characteristically large irrigation and vessel permeability in tumors, nanoparticles display a high tendency to 


\section{WILEY-VCH}

accumulate in cancer tissue. However, the same holds true for healthy liver and spleen tissue. Andreou et al. used this usually undesired effect to their advantage, to achieve high resolution in vivo imaging of certain tumors. ${ }^{[167]}$ For this purpose, Raman-tagged $\mathrm{Au} / \mathrm{SiO}_{2}$ nanoparticles were injected into mice with liver cancer, allowing a precise identification of the tumor limits by SERS imaging and, consequently, a less invasive clinical ablation of the damaged tissue.

One of the most appealing aspects of nanomedicine is that active targeting can be achieved by functionalization of the nanoparticles with molecules such as proteins or aptamers, which show high affinity for receptors that are typically overexpressed by cancer cells. ${ }^{[168]}$ As a strategy to suppress drug leakage during transport, Zhang et al. used an aptamer to block the pores of drugloaded particles. Since the aptamer selectively recognizes a molecule that is exclusively present inside the cytosol, the drug would only be released by endocytosed particles. ${ }^{[74]}$ In a related work, Liu et al. used mesoporous silica-coated AuNRs loaded with doxorubicin and chose a biocompatible material that melts at $39^{\circ} \mathrm{C}$ as the gatekeeper, whereby the nanoparticles were tagged with folate moieties to specifically target KB cancer cells. ${ }^{[71]}$ NIR excitation would then trigger both the release of the chemotherapy drug and hyperthermal treatment.

Xia et al. used AuNRs coated with a silica shell containing entrapped quantum dots, and further functionalized with folic acid to improve cancer cell targeting. ${ }^{[65]}$ In this system, the quantum dots allowed fluorescence imaging, whereas the AuNRs could be monitored by X-ray Computed Tomography (CT) while enabling photothermal treatment when illuminated with NIR light. Furthermore, photothermal and photodynamic therapies can be combined by fitting the particles with a photosensitizer capable of generating cytotoxic oxygen species that promote cell ablation. ${ }^{[169]}$ For such applications, Zhang et al. used mesoporous silica-coated gold nanorods loaded with a photodynamic agent. ${ }^{[74]}$ The particles were functionalized with an aptamer to recognize a molecule in the nucleolus, and the silica pores were capped with silver particles, thereby allowing release of the cargo upon contact with glutathione inside the cytosol. 


\section{WILEY-VCH}

\subsection{Catalysis}

\subsubsection{Noble Metal Nanoparticle Catalysis}

Few inventions have impacted modern chemistry as profoundly as the discovery of noble metal catalysis in the early $19^{\text {th }}$ century. During the last two decades the field of heterogeneous catalysis has witnessed a shift of research interest toward the utilization of nanoparticles as active components for green catalysis. ${ }^{[170]}$ Compared to classic bulk metal catalysts, materials based on nanoparticles promise greatly improved efficacies due to the combination of a huge internal surface area, the high reactivity of curved metal surfaces that arises from strain-induced lattice distortions as well as from the presence of electronically unsaturated interfacial atoms. ${ }^{[59,114]}$ This activity increase by size reduction is particularly evident for ultrasmall nanoparticles, as shown in Figure $\mathbf{1 3}$ for the case of $\mathrm{CO}$ oxidation catalyzed by silica-coated AuNPs with diameters between 1.5 and $2.3 \mathrm{~nm}$. The same trend was found in studies of the turnover frequency (TOF, i.e., the moles of product obtained per moles of accessible catalyst atoms) in the Au-catalyzed reduction of $\mathrm{p}$-nitrophenol by $\mathrm{NaBH}_{4}$ for small cores with diameters between 2.8 and $4.5 \mathrm{~nm}$ as well as for larger spheres with diameters of 21-50 nm and 43-104 nm. ${ }^{[104,171,172]}$ Thereby, the presence of a protecting shell proved vital for the long-term stability of the catalysts, preventing both colloidal aggregation in solution and fusion of the nanoparticles during high temperature reactions, which would lead to a minimization of the total particle surface energy accompanied by a loss of most catalytically active sites. Equally important parameters are the core surface functionality and shell porosity, which were reported to have a significant impact on the catalytic efficiency of AuNPs. ${ }^{[15,106]}$ 


\section{WILEY-VCH}
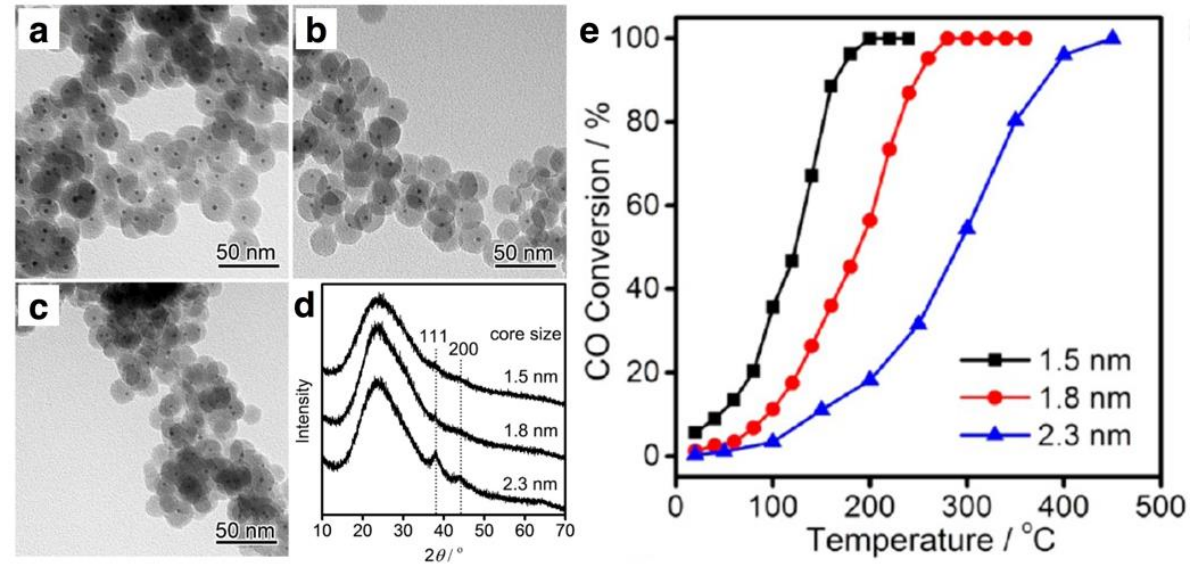

Figure 13. High temperature $\mathrm{CO}$ oxidation catalyzed by $\mathrm{Au} / \mathrm{mSiO}_{2}$ nanoparticles with ultrasmall cores. a,b,c) TEM micrographs of hybrid particles annealed at $500{ }^{\circ} \mathrm{C}$ with Au cores of $2.3,1.8$, and $1.5 \mathrm{~nm}$ diameter, respectively. d) XRD patterns of the temperature treated coreshell particles, showing a large peak near $23^{\circ}$ resulting from the amorphous silica shell, as well smaller peaks attributable to the $\{111\}$ and $\{200\}$ diffractions of gold. e) CO conversion plotted as a function of the reaction temperature, for different core sizes measured with identical amounts of catalyst (including the silica shell). For 100\% conversion a temperature around $180{ }^{\circ} \mathrm{C}$ was necessary for $1.5 \mathrm{~nm}$ cores, whereas $1.8 \mathrm{~nm}$ and $2.3 \mathrm{~nm}$ particles required about $260{ }^{\circ} \mathrm{C}$ and $400{ }^{\circ} \mathrm{C}$, respectively. Adapted under the terms of the ACS AuthorChoice license. ${ }^{[114]}$ Copyright 2014, American Chemical Society. Permission requests for the material excerpted should be directed to the ACS. (http://pubs.acs.org/doi/abs/10.1021\%2Fnn502349k)

\subsubsection{Catalyst Stability Improvement by Encapsulation}

Coating with porous metal oxides has opened avenues for the realization of highly efficient, reusable catalysts and is currently a standard practice for the synthesis of nanoparticle-based catalysts. ${ }^{[173]}$ Important early examples for this design principle are $\mathrm{Pd} / \mathrm{mSiO}_{2}$ and $\mathrm{Pt} / \mathrm{mSiO}_{2}$ catalysts, which allowed efficient acetylene and ethylene hydrogenation and were successfully employed for high-temperature $\mathrm{CO}$ oxidation without significant reactivity losses, even after heating up to $750{ }^{\circ} \mathrm{C}$ in air. ${ }^{[21,22]}$ Lately, there has been interest not only in preventing nanoparticle sintering, but also in controlling it toward the creation of nanoalloys. High temperature alloying was recently demonstrated for silica-coated $\mathrm{Au}-\mathrm{Ag}, \mathrm{Au}-\mathrm{Pd}$, and $\mathrm{Au}-\mathrm{Pt}$ particles. ${ }^{[24]}$ Additionally, Ge et al. reported the fabrication of bimetallic $\mathrm{CuNi} / \mathrm{mSiO}_{2}$ catalysts that displayed a low activation energy in the reduction of p-nitrophenol with $\mathrm{NaBH}_{4}{ }^{[174]}$ This was achieved by a series of oxidative and reductive treatment steps of silica-coated aggregates containing $\mathrm{Cu}$ and $\mathrm{Ni}$ nanoparticles. The shell porosity necessary for reactant exchange may be 


\section{WILEY-VCH}

an intrinsic property of certain thin silica shells ${ }^{[105]}$ but can also be introduced post-synthetically into thicker shells via etching with $\mathrm{NaOH} \cdot{ }^{[175,176]}$ However, these approaches lack precise control over the pore dimensions and are not easily applicable to hydrolysis resistant metal oxides such as $\mathrm{TiO}_{2}$, which are frequently used for catalyst encapsulation. As an alternative, as described above, soft templating methods using micelle building surfactants or amphiphilic block-copolymers have granted access to noble metal core-shell catalysts protected with mesoporous layers of $\mathrm{SiO}_{2}, \mathrm{TiO}_{2}, \mathrm{ZrO}_{2}, \mathrm{CeO}_{2}$, or $\mathrm{SnO}_{2} \cdot{ }^{[173]}$ Removal of the typical scaffold surfactants like TTAB or CTAB can be accomplished by either calcination or simply washing with $\mathrm{HCl}^{[14]}$

\subsubsection{Yolk-Shell Nanoreactors}

Yolk-shell composite particles, in which the active metal core is not covalently connected to the shell material but instead able to move freely inside the protective hull, can be regarded as a further development of core-shell catalysts. ${ }^{[59]}$ The obvious advantage of such a nanoreactor design lies in the increased active surface area accessible to the reactants and the resulting higher TOFs, as compared to particles with direct core-shell contact. ${ }^{[172]}$ Figure 14 displays an example of such yolk-shell particles, prepared by etching encapsulated, oversized core particles to the desired size. This strategy was first employed by Lee et al. for studies of the core-size dependent catalytic activity. ${ }^{[172]}$ The authors used $\mathrm{KCN}$ to etch $120 \mathrm{~nm}$ sized silica-coated gold nanospheres down to $43 \mathrm{~nm}$, thereby enhancing the average catalyst TOF in the reduction of $\mathrm{p}$ nitrophenol by a factor of nearly 5 , which was partially attributed to a higher edge roughness of the cores. The same group later presented a similar approach for the preparation of $\mathrm{Ni} / \mathrm{mSiO} \mathrm{O}_{2}$ yolk-shell particles by $\mathrm{HCl}$-induced etching and employed these sintering resistant nanoreactors to catalyze the steam methane reforming reaction. ${ }^{[23]} \mathrm{An}$ interesting route to metal/ $\mathrm{SiO}_{2}$ yolkshell particles that does not rely on core etching, i.e. avoiding the partial destruction of catalytic material, was introduced by Wang et al. using PS-P4VP microspheres to immobilize small $\mathrm{Au}$ nanoparticles that were sintered into a single particle after coating with a mesoporous silica 


\section{WILEY-VCH}

shell and utilized for the catalytic reduction of p-nitrophenol. ${ }^{[171]}$ Similar architectures were obtained by silica condensation on gelatin-coated metal nanoparticles, by utilizing oil-in-water miniemulsions as a template for the one-pot synthesis of encapsulated clusters, or by inside-out etching of silica shells synthesized in the presence of (3-aminopropyl) trimethoxysilane. ${ }^{[104,119,177,178]}$ In a different approach, Sanles-Sobrido et al. produced hollow silica shells with an interior Pt/Ni cladding by first mobilizing dendritic PtNPs on polystyrene (PS) colloids, followed by silica coating, stripping the PS cores with an $\mathrm{EtOH} / \mathrm{CHCl}_{3}$ mixture, and metal-catalyzed Ni deposition over the enclosed Pt seeds. ${ }^{[179]}$ Besides its primary function as a semi-permeable barrier, the silica layer of both core-shell and yolk-shell particles could also be used to template new nanoreactor types by overgrowth with another metal-oxide layer like $\mathrm{TiO}_{2}, \mathrm{SnO}_{2}, \mathrm{ZrO}_{2}$, or $\mathrm{CeO}_{2}$ and subsequent hydrolysis of the sacrificial silica layer. Besides the improved hydrothermal stability of these shell materials, several papers have reported enhanced catalytic performance due to synergistic effects for certain metal/metal oxide combinations such as $\mathrm{Au} / \mathrm{TiO}_{2}$ or $\mathrm{Pd} / \mathrm{CeO}_{2}$ providing a smart design scheme for new, highly efficient nanocatalysts. ${ }^{[59,173]}$

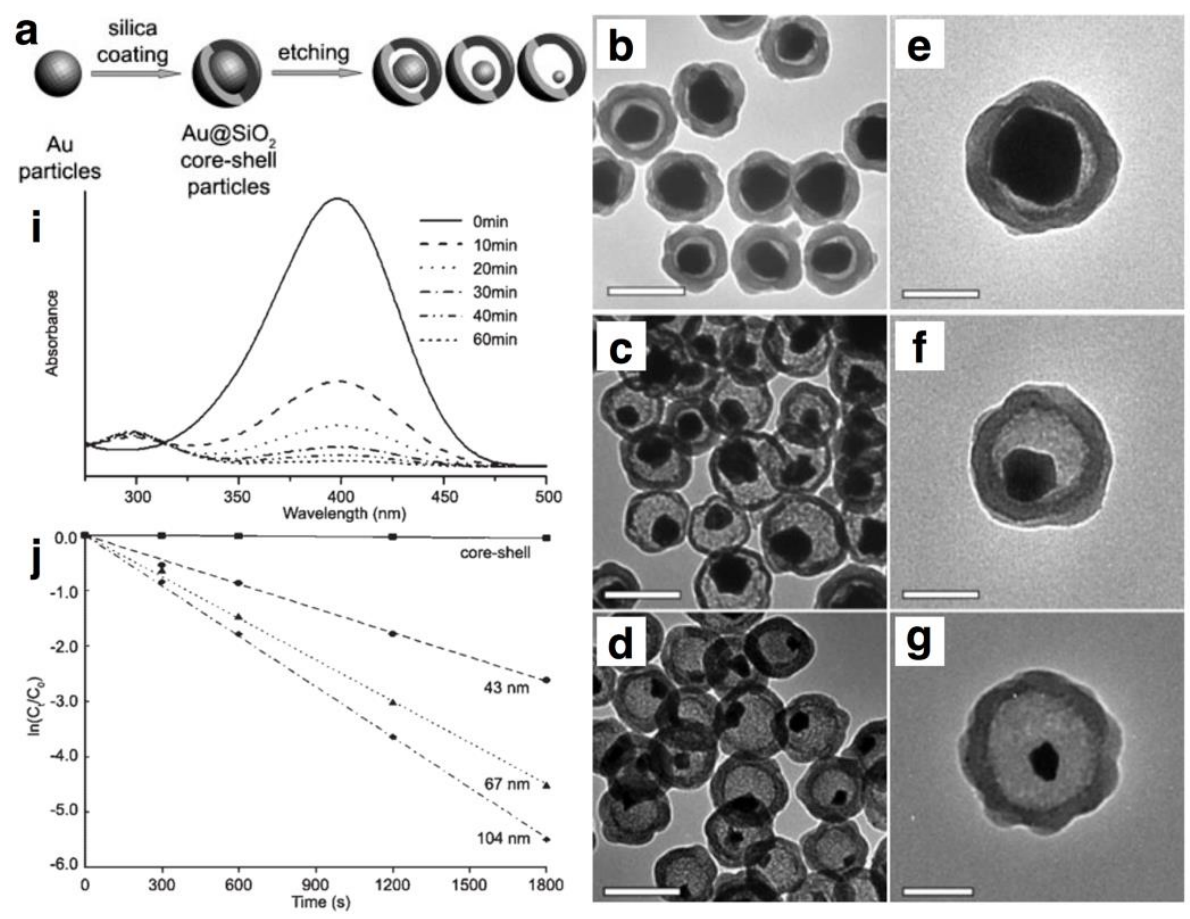




\section{WILEY-VCH}

Figure 14. Yolk-shell nanoreactors for the catalytic reduction of p-nitrophenol by $\mathrm{NaBH}_{4}$. a) Synthesis of nanorattles via partial etching of gold cores with KCN. b-d) TEM micrographs of the resulting particles with cores of 104, 67, $43 \mathrm{~nm}$ diameter from top to bottom (scale bars: $200 \mathrm{~nm}$ ). e-g) The same particles at higher magnification (scale bars: $100 \mathrm{~nm}$ ). h) UV/vis monitoring of the reduction reaction in the presence of yolk-shell particles with $104 \mathrm{~nm} \mathrm{Au}$ cores. i) Plots of $\ln \left(\mathrm{C}_{t} / \mathrm{C}_{0}\right)$ vs. reaction time for core-shell and yolk-shell particles extracted from the corresponding time-dependent UV/vis measurements: the faster decay for the larger particles was related to the higher total amount of gold surface as the particle number concentration was held constant. In contrast, calculated TOFs normalized to the available catalyst surface showed a 5-fold increase when the core size was decreased from 104 to $43 \mathrm{~nm}$, which was attributed to sharper edges. Adapted with permission. ${ }^{[172]}$ Copyright 2013, WileyVCH Verlag GmbH \& Co. KGaA, Weinheim.

\subsubsection{Multifunctional Composite Catalysts}

Certain applications in catalysis profit from even more complex nanoparticle architectures. For heterogeneous catalysis in dispersion, magnetic properties offer a distinct advantage since they readily allow the recollection of nanoparticles, thereby improving the recyclability of the catalyst. ${ }^{[180,181]}$ Ge et al. managed to combine high catalytic activity, steric protection, and a magnetic response in one composite particle by adsorption of Au clusters onto ferromagnetic $\mathrm{Fe}_{3} \mathrm{O}_{4}$ cores and subsequent coating with a mesoporous silica shell, demonstrating efficient reuse up to six catalysis cycles. ${ }^{[175]}$ Going one step further, noble metal/mesoporous shell composites tailored for plasmon-enhanced photocatalysis offer intriguing possibilities to control the catalytic efficiency by taking advantage of the local field enhancement under light irradiation near the LSPR. This effect was demonstrated for nanocomposites of CdS quantum dots attached to silica-coated gold cores as displayed in Figure 15. The silica layer served here as both an electrical insulator and a spacer, providing control over the photocatalytic hydrogen evolution reaction. ${ }^{[182]}$ 

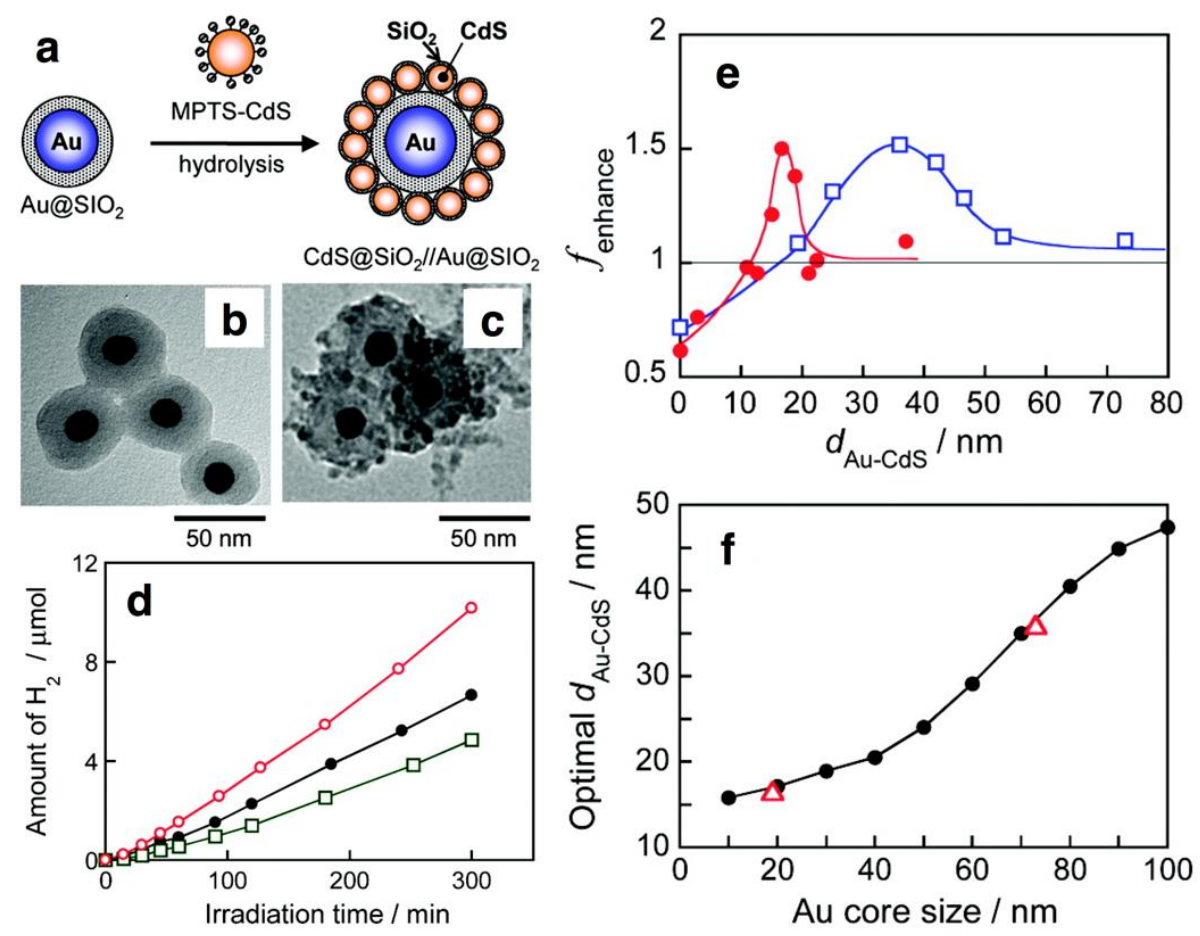

Figure 15. Plasmon-enhanced photocatalysis of $\mathrm{Au} / \mathrm{SiO}_{2}-\mathrm{CdS} / \mathrm{SiO}_{2}$ heterocomposites. a) Scheme of the coupling of $\mathrm{CdS}$ quantum dots to silica-coated AuNSs: the 3mercaptopropyltrimethoxysilane (MPTS) capped QDs were first allowed to adsorb onto the plasmonic carrier particles in a toluene/isopropanol mixture and then coated with a thin silica layer via water-induced hydrolysis of the ligand at $90{ }^{\circ} \mathrm{C}$. b,c) TEM micrographs of $\mathrm{Au} / \mathrm{SiO}_{2}$ particles before (left) and after (right) adsorption of $\mathrm{CdS} / \mathrm{SiO}_{2}$ particles. d) Kinetics of hydrogen evolution under illumination with a Xe-lamp for $\mathrm{CdS} / \mathrm{SiO}_{2}$ particles (black, closed circles) and $\mathrm{Au} / \mathrm{SiO}_{2}-\mathrm{CdS} / \mathrm{SiO}_{2}$ heterocomposites with $2.8 \mathrm{~nm}$ (green, open squares) and $17 \mathrm{~nm}$ (red, closed circles) silica shells on comparable $\mathrm{Au}$ cores. The data were obtained with identical concentrations of $\mathrm{CdS}$ and $\mathrm{Rh}$ cocatalyst, to which electrons of the photoexcited QDs are eventually transferred, inducing the reduction of $\mathrm{H}^{+}$ions: whereas binding to Au cores with the short spacer led to a decrease of the $\mathrm{H}_{2}$ evolution rate compared to bare $\mathrm{CdS}$, employing gold particles with a thicker shell improved the photocatalytic activity. e) Enhancement factors of the heterocomposites compared to bare QDs, as a function of interparticle spacing for $19 \mathrm{~nm}$ (red, closed circles) and $73 \mathrm{~nm}$ (blue, open squares) Au cores. f) Modelled (black, solid circles) and measured (red, open triangles) ideal spacings for highest enhancement depending on $\mathrm{Au}$ particle size. Adapted with permission. ${ }^{[182]}$ Copyright 2011, American Chemical Society.

Another recent example of plasmon-enhanced photocatalysis was presented by Zhu et al., who investigated the activity of various anisotropic, bimetallic Au-Pd-nanostructures synthesized by site-selective silica protection of the gold cores. ${ }^{[58]}$ As shown in Figure 16, higher conversion in a Suzuki coupling reaction could be achieved when the electric near-field was concentrated around the catalytically active sites, i.e. Pd. Such synergistic effects arising in the proximity of noble metal cores, metal oxide shells, and other catalytically active components, constitute an 
interesting line for further research, and detailed understanding of the interplay of all materials involved will be necessary to access the full potential of nanocomposites for catalysis.
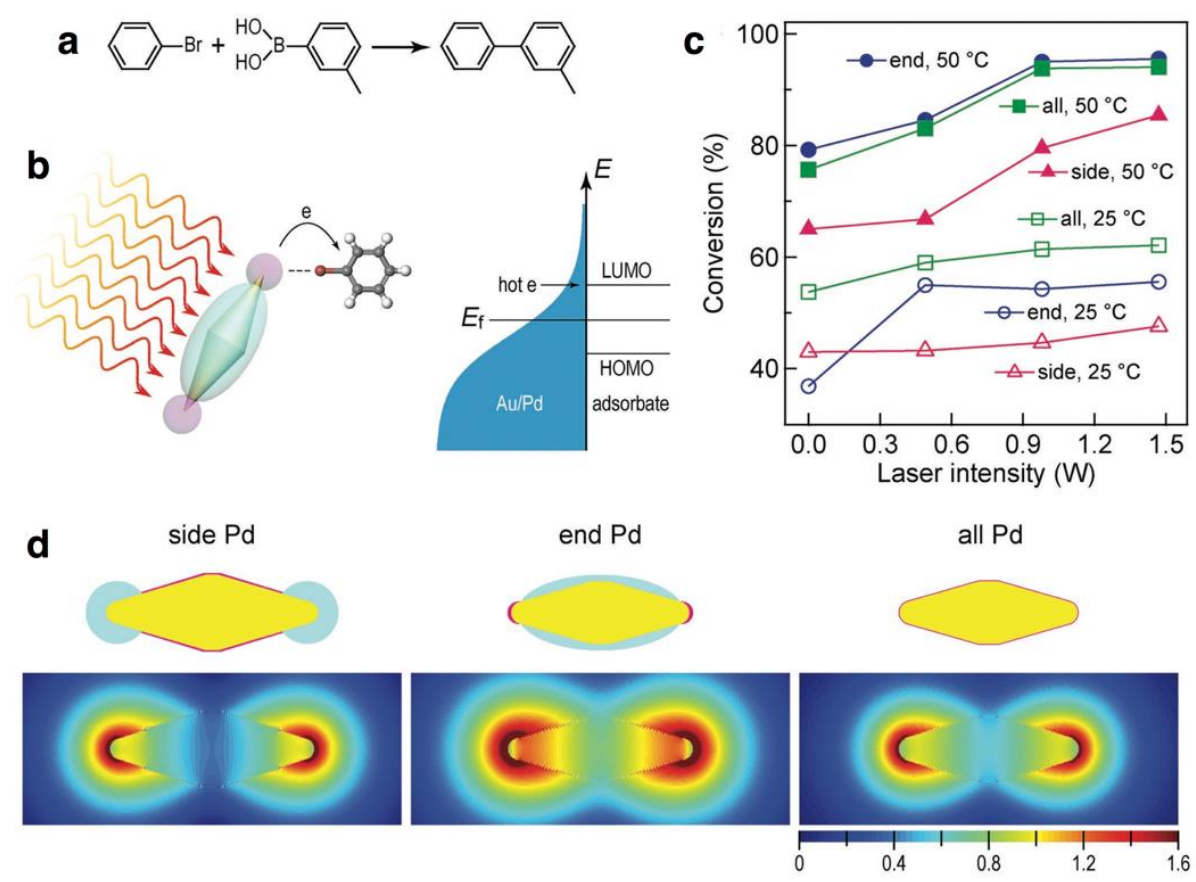

Figure 16. Plasmon-enhanced photocatalysis of a Suzuki coupling reaction in the presence of (partially) Pd-coated $\mathrm{Au} / \mathrm{SiO}_{2}$ nanobipyramids. a,b) Reaction between bromobenzene and $\mathrm{m}$ tolylboronic acid and schematic view of the mechanism: hot electrons created by the bipyramid acting as a plasmonic antenna are injected into the lowest unoccupied orbital of bromobenzene, exciting the adsorbate and promoting cleavage of the $\mathrm{C}$-Br bond. As this is the rate determining step, the catalytic efficiency is connected to the ability of the composite particles to absorb light and transfer the energy provided by laser irradiation at $808 \mathrm{~nm}$, i.e., close to the longitudinal LSPR of the metal core. c) Comparison of the conversion achieved with bipyramids coated with $\mathrm{Pd}$ on different sites obtained at 25 and $50{ }^{\circ} \mathrm{C}$. d) Electric near-field enhancement contour plots for side-, end-, and fully Pd-coated bipyramids. Optimal catalytic efficiency was achieved for nanoparticles having either a full Pd coating or Pd at the ends, where the strongest optical fields were excited. Adapted with permission. ${ }^{[58]}$ Copyright 2013, Wiley VCH Verlag GmbH \& Co. KGaA, Weinheim.

\subsection{Photovoltaics}

\subsubsection{Plasmon-Enhanced Photovoltaics}

Integration of plasmonic nanoparticles into photovoltaic devices has seen great progress during the last decade and is regarded crucial especially for the design of highly efficient thin film solar cells. ${ }^{[183]}$ This holds true for organic solar cells (OSCs) based on semiconducting polymers or polymer/fullerene mixtures, as much as for (solid state) dye-sensitized solar cells (DSSCs) 


\section{WILEY-VCH}

and for the recently developed perovskite based photovoltaic cells (PSCs). ${ }^{[184,185]}$ Whereas these solar cell types promise advantages over conventional silicon-based designs in terms of material costs, low temperature solution-processing, and applicability to flexible substrates, they all face a common challenge: the charge carrier (i.e., electron and hole) mobilities inside the employed materials are rather low, and therefore efficient photocurrent generation takes place only in the proximity to donor-acceptor interfaces. ${ }^{[186]}$ Due to this physical constraint, the maximum thickness of the active layer is usually found in the range of tens of nanometers for OSCs, ${ }^{[186]}$ several hundred nanometers for PSCs, ${ }^{[187]}$ and about two micrometers for solid-state DSSCs. ${ }^{[49]}$ In each case, this is too small to achieve complete absorption of the incident light over the entire solar spectrum with single-junction devices. To harvest a major fraction of the incoming photon flux in such thin films, light management with subwavelength-sized structures that do not disturb the film morphologies but still interact strongly with visible and near infrared light at low volume fractions is necessary. Consequently, a lot of research activity has been dedicated to the implementation of silica-coated $\mathrm{Au}, \mathrm{Ag}, \mathrm{Cu}$, and $\mathrm{Al}$ nanoparticles into solar cells. ${ }^{[188]}$ Such particles generally lead to enhancements of the external quantum efficiency (EQE) over a relatively broad spectral range and consequently improve their overall power conversion efficiency (PCE).

\subsubsection{Integration of Plasmonic Nanoparticles into Solar Cells}

Coating the metal cores with a chemically and electronically insulating silica layer is required to different extents, depending on the position of the plasmonic particles within the layer structure of the cells. Typically, when the particles are in contact with the active layer, silica shells of 3-10 nm are used for insulation and their thickness is known to critically influence the interaction of the particles with the photocurrent generating medium. ${ }^{[52,185]}$ Besides obvious oxidation issues, the main reason for encapsulating plasmonic particles is related to preventing the recombination of charge carriers promoted by the metal surface, i.e., exciton quenching. ${ }^{[50]}$ Apart from electronic shielding, the shell may serve to suppress energy transfer from excited 


\section{WILEY-VCH}

molecules of the active medium into the nanoparticles surface plasmon while offering protection against corrosion by the cell medium or particle fusion during thermal processing. ${ }^{[49]}$ The important role of an insulation shell for certain cell architectures was demonstrated by Choi et al. for silver nanoparticles integrated into PTB7:PC70BM-based polymer solar cells. ${ }^{[50]}$ The authors found that bare AgNS provided enhancement only when located outside the active layer, otherwise leading to a strong deterioration of the cell performance. Coating the particles with $10 \mathrm{~nm}$ thick silica shells solved this problem, allowing partial incorporation of $\mathrm{Ag} / \mathrm{SiO}_{2}$ into the current generating polymer matrix (see Figure 17). Whereas completely embedding the silver particles inside the electron blocking layer of the cells yielded a PCE increase of 10\%, locating $\mathrm{Ag} / \mathrm{SiO}_{2}$ hybrid particles at the interface between the electron blocking and the active layers gave rise to a $19 \%$ improvement.
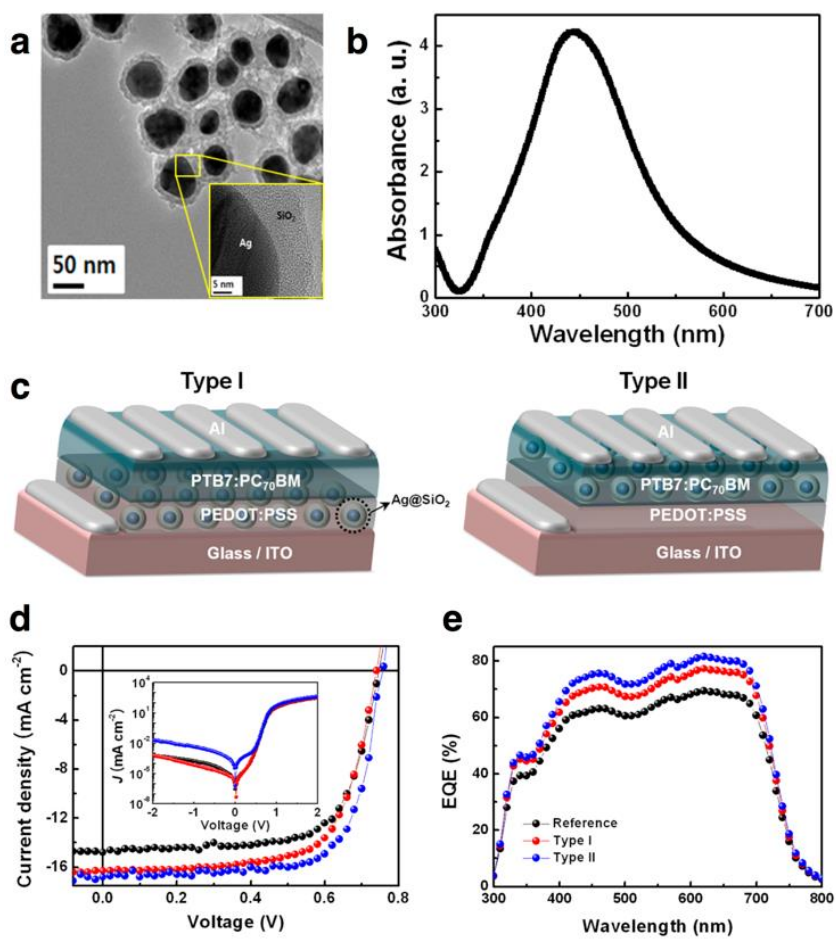

Figure 17. Integration of $\mathrm{Ag} / \mathrm{SiO}_{2}$ nanoparticles into polymer solar cells. a) TEM micrographs of AgNPs with a diameter of approximately $50 \mathrm{~nm}$ and a $10 \mathrm{~nm}$ thick amorphous silica coating. b) UV/vis spectrum of the core-shell particles dispersed in EtOH. c) Illustration of two investigated device structures: in Type I solar cells the plasmonic particles were embedded in the electron blocking PEDOT/PSS layer, whereas for the Type II architecture the $\mathrm{Ag} / \mathrm{SiO}_{2}$ particles were located at the interface between PEDOT/PSS and the active layer. d) Dark 
current-voltage curves (I-V curves) demonstrated a clear improvement of the short circuit voltage for Type I and II cells, as compared to reference cells without particles. e) Wavelengthdependent measurements of the EQE for the three cell types showed enhancement between 400 and $700 \mathrm{~nm}$ for both plasmonic cell types. Integration of the plasmonic particles into the active layer (Type II) was reported to result in a significantly improved enhancement factor, but strictly required the presence of the insulating silica shell. Adapted with permission. ${ }^{[50]}$ Copyright 2013, American Chemical Society.

\subsubsection{Plasmonic Enhancement Effects}

Having established that use of noble metal nanoparticles in photovoltaics requires protection by an insulating shell, we now turn to discussing the physical effects behind plasmonic light absorption enhancement in thin film solar cells. Efficiency enhancements, due to plasmonic effects are generally attributed to radiant and non-radiant effects, as illustrated in Figure $\mathbf{1 8}$ and explained in more detail in Ref. ${ }^{[185]}$ In what follows, emphasis is put on explaining the implications for the design of core-shell morphologies that enable an optimal utilization of each of these plasmonic effects.

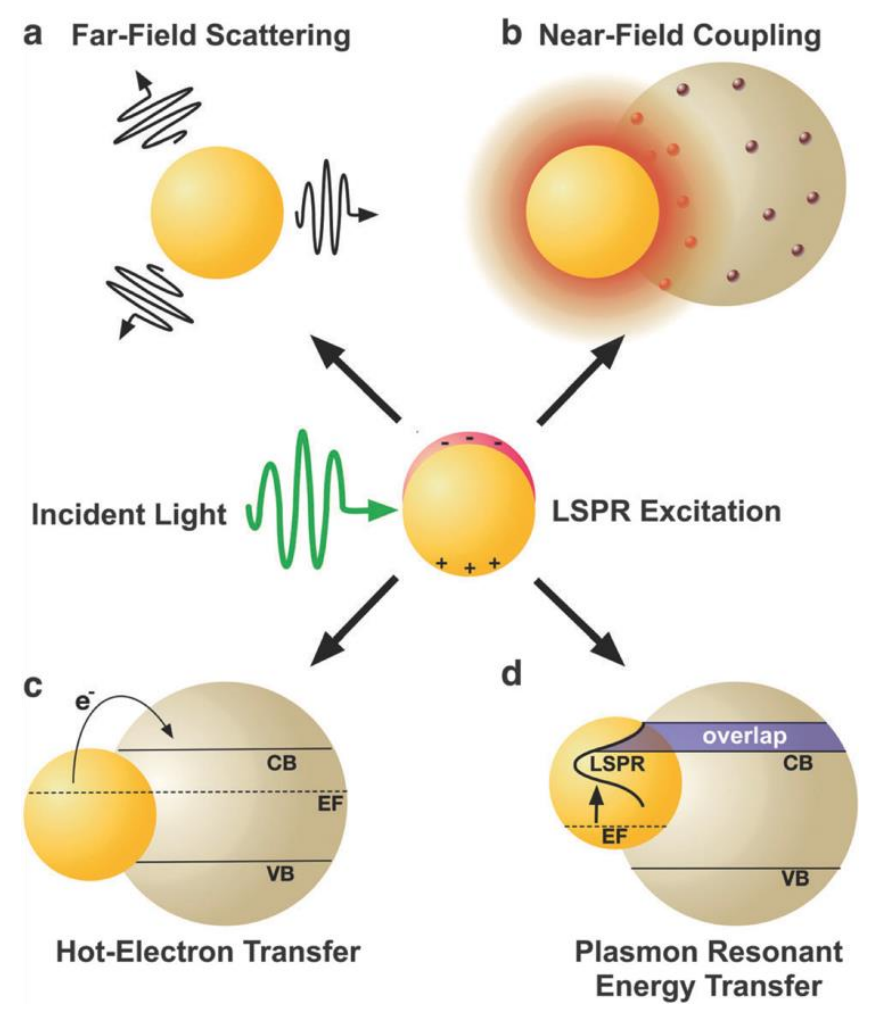

Figure 18. Illustration of the most prominent plasmonic enhancement mechanisms in solar cells containing noble metal nanoparticles. After excitation by light irradiation, the energy stored in the surface plasmon can be transferred from the particle LSPR to the current generating material, 


\section{WILEY-VCH}

either by radiative $(\mathrm{a}, \mathrm{b})$ or non-radiative effects $(\mathrm{c}, \mathrm{d})$. a) Far-field scattering, i.e. re-irradiation of electromagnetic waves dominates for large particles of low-loss noble metals and can increase the pathlength of incident photons by multiples of the active layer thickness. b) Nearfield coupling can become the major contribution to enhancement if the metal particles are at least partially located in the active layer and surrounded by very thin shells. c) Hot electron transfer, i.e., energy transfer via injection of electrons from the excited plasmonic particle into the conduction band of a nearby semiconductor, occurs mostly for lossy metals coated with shells thinner than $2 \mathrm{~nm}$. d) Plasmon resonant energy transfer (PRET) requires spectral overlap between the LSPR and semiconductor material located within the particle near-field. Reproduced under the terms of the CC BY 3.0 license. ${ }^{[185]}$ Copyright 2016, published by The Royal Society of Chemistry. (http://dx.doi.org/10.1039/C5EE03847B)

Surface plasmons are very efficiently excited by the electric field of incident light with a frequency close the particle specific LSPR. ${ }^{[2]}$ The energy stored in a surface plasmon can be infused into the photocurrent generating medium either by re-emission into the near-field and far-field of the embedded particle or by absorption and subsequent non-radiative transfer processes. ${ }^{[185]}$ With increasing particle size, the ratio of scattered to absorbed light increases, whereby the scattering cross-section of a plasmonic particle can easily surpass its geometrical cross-section by over an order of magnitude, thus resulting in significant enhancement effects, even at low volume fractions. ${ }^{[186]}$ Far-field scattering can be used either to couple light into layers with a permittivity higher than that of air by placing the nanostructures on top of the cell, or to extend the light paths inside the active material, by incorporating core-shell particles into the layer structures. ${ }^{[183,189]}$ Also, in contrast to other effects used to enhance solar cell performance, far-field scattering does not rely on a precise adjustment of the silica shell thickness.

Near-field enhancement due to a concentration of the electromagnetic field close to the particle surface has been identified as one of the most important reasons for PCE enhancement in cells in which the plasmonic cores are surrounded by very thin shells between $2-10 \mathrm{~nm}$ and partially or completely embedded inside the active layer. ${ }^{[51,52]}$ The role of radiative enhancement has recently been studied for different materials, particle geometries, and coating thicknesses by Carretero-Palacios et al. for the case of perovskite cells, using FDTD simulations. The authors 


\section{WILEY-VCH}

concluded that $\mathrm{Ag}$ particles with diameters above $90 \mathrm{~nm}$ and silica shells thinner than $10 \mathrm{~nm}$ were more efficient enhancers than comparable Au or Al particles, whereby edge sharpness was found to play a minor role as the local field enhancement for stars and cubes, known as the lightning rod effect, did not lead to significant enhancement of the overall field in the volume around the particles, compared to large spheres. ${ }^{[52]}$

For optimal radiative enhancement in general, the particle LSPR should be located in a spectral region in which the current generating material itself displays weak absorption (i.e., close to the semiconductor band edge normally located in the red or NIR). ${ }^{[92,186,190]}$ Jankovich et al. demonstrated the influence of spectral matching for OSCs, by combining polymers with band edges around $700 \mathrm{~nm}\left(\mathrm{P} 3 \mathrm{HT}: \mathrm{PC}_{60} \mathrm{BM}\right)$ and $860 \mathrm{~nm}$ (PBDTT-DPP:PC $\left.{ }_{60} \mathrm{BM}\right)$, respectively, and silica-coated gold nanoparticles of varying anisotropy displaying LSPRs between $540 \mathrm{~nm}$ and 830 nm. ${ }^{[186]}$ As shown Figure 19, enhancements of the PCE were clearly linked to the LSPR position of the particles, delivering optimal results when matched to the band edge of the active layer material. 
a
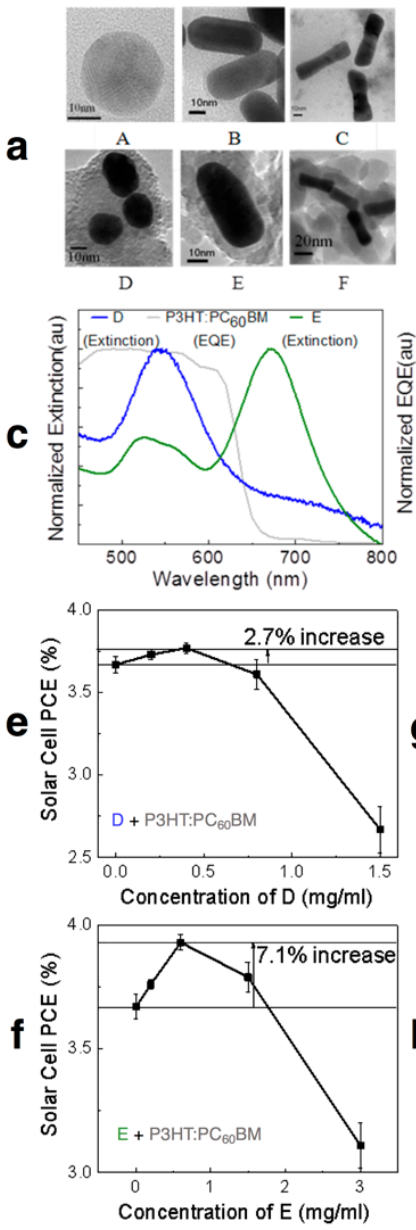

b
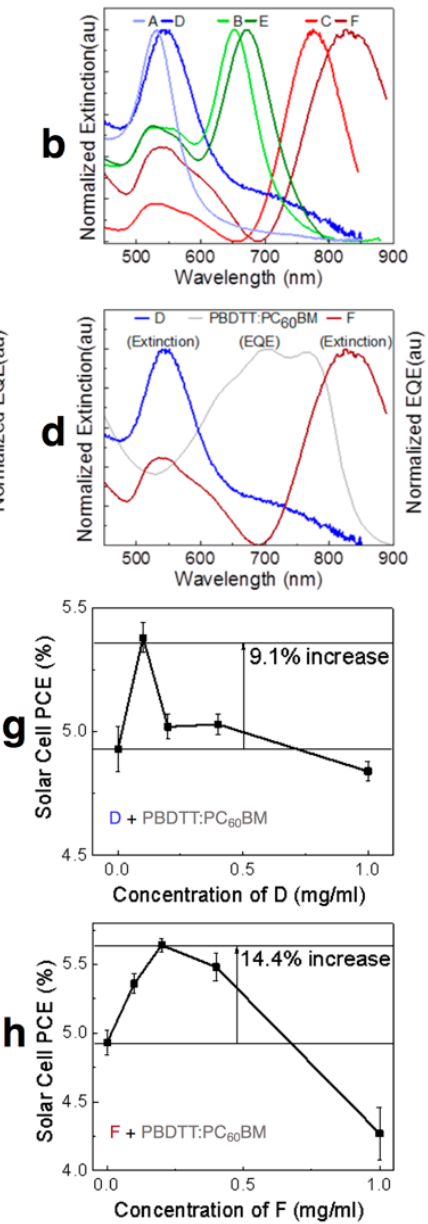

Figure 19. Adjusting the optical properties of $\mathrm{Au} / \mathrm{SiO}_{2}$ core-shell particles for optimal plasmonic enhancement of polymer solar cells with different band edges. a) TEM micrographs of bare and silica-encapsulated gold nanospheres and rods of different aspect ratios $(\sim 2.5$ for $\mathrm{B}, \mathrm{E} ; \sim 4.0$ for $\mathrm{C}, \mathrm{F})$. b) Corresponding extinction spectra in water: for all particles the silica shell leads to a slight LSPR red shift due to a local increase in refractive index. For the coated particles, LSPRs around $540 \mathrm{~nm}$ (AuNSs), $670 \mathrm{~nm}$ (AuNRs-AR2.5), and $830 \mathrm{~nm}$ (AuNRsAR4) were measured. c,d) Comparison of the $\mathrm{Au} / \mathrm{SiO}_{2}$ particle extinction spectra with wavelength-dependent EQE measurements of the active layer materials (P3HT:PC ${ }_{60} \mathrm{BM}$ and PBDTT:PC $\left.{ }_{60} \mathrm{BM}\right)$. e,f) Particle concentration-dependent PCEs for P3HT:PC ${ }_{60} \mathrm{BM}$-based devices loaded with silica-coated AuNSs or AuNR-AR2.5. g,h) Particle concentrationdependent PCEs for PBDTT:PC ${ }_{60}$ BM-based cells with silica-coated AuNSs or AuNR-AR4. The highest enhancements were achieved by combining active layer materials and particles with complementary optical properties (f, $g, h$ ). Reduction of the solar cell PCEs at high particle concentrations were attributed to changes in the layer morphology. Adapted with permission. ${ }^{[186]}$ Copyright 2013, American Chemical Society.

In contrast to radiative effects, in which the particles basically act as new light sources with similar emission properties as the incident radiation, energy transfer via plasmon resonant energy transfer (PRET) and hot-electron injection can be used to harvest photons with energies below the band edge of the active layer material. ${ }^{[185]}$ Energy transfer by PRET is possible if 


\section{WILEY-VCH}

there is spectral overlap between the surface plasmon of an electrically isolated particle and the absorption band of a semiconductor material located in its near-field. ${ }^{[185]}$ Hereby, energy transfer from the plasmonic core to the active layer material occurs by dipole-dipole coupling and is most efficient for silica shell thicknesses below $10 \mathrm{~nm} \cdot{ }^{[191]}$ The occurrence of hot electrons during plasmon relaxation is most common for absorbing materials like $\mathrm{Au}$, which displays strong Ohmic losses due to d-band transitions about $2.3 \mathrm{eV}$ below the Fermi level. ${ }^{[192]}$ In the presence of a very thin insulator forming a Schottky barrier between the metal surface and an adjacent semiconductor, these electrons can be harvested. Hot electron injection was reported to be most relevant for solar cell enhancement in the case of nanoparticles with diameters below $20 \mathrm{~nm}$ and silica shells less than 2 nm thick. ${ }^{[185,193]}$

Apart from these mechanisms, leading indirectly or directly to energy transfer from nanoparticles to the photoactive material, several publications have concluded that the presence of noble metal/silica core-shell particles can influence the overall electrical properties of the cell. For example, Zhang et al. concluded from photoluminescence measurements that by embedding $\mathrm{Au} / \mathrm{SiO}_{2}$ particles the exciton binding energy in perovskite cells could be lowered and thus charge carrier separation would be accelerated. ${ }^{[48]}$ Further, by incorporating $\mathrm{Au} @ \mathrm{SiO}_{2}$ nanorods with a longitudinal LSPR around $700 \mathrm{~nm}$ and a $9.5 \mathrm{~nm}$ thick silica shell at the interface between the hole transport and the active layers of a PEDOT:PSS/ $\mathrm{CH}_{3} \mathrm{NH}_{3} \mathrm{PbI}_{3} / \mathrm{PCBM} / \mathrm{Al}$ heterojunction perovskite solar cell, Wu et al. found an average conversion efficiency gain of more than $40 \%$, reaching a maximum PCE of $17.6 \%$, which was ascribed to a combination of plasmonic absorption enhancement and improved exciton recombination resistance, as shown by electrochemical impedance spectroscopy measurements. ${ }^{[194]}$ More recently, Ye et al. presented a study of the plasmonic enhancement of tri-cation and dual-anion mixed perovskite solar cell performance by gold nanospheres coated with a $15 \mathrm{~nm}$ thick silica layer, reporting a $29 \%$ efficiency gain. Apart from the reportedly higher structural and thermal stability of mixed perovskites, this work is interesting because it demonstrated via time-of-flight 


\section{WILEY-VCH}

photoconductivity measurements that plasmonic particles can increase the hole mobility by nearly one order of magnitude and the electron mobility by almost two orders of magnitude, as shown in Figure 20. The resulting match in charge carrier mobility, as well as lifetime enhancements and enhanced light absorption, were made responsible for the high maximum PCE of $20.3 \%$ achieved in this work. ${ }^{[187]}$
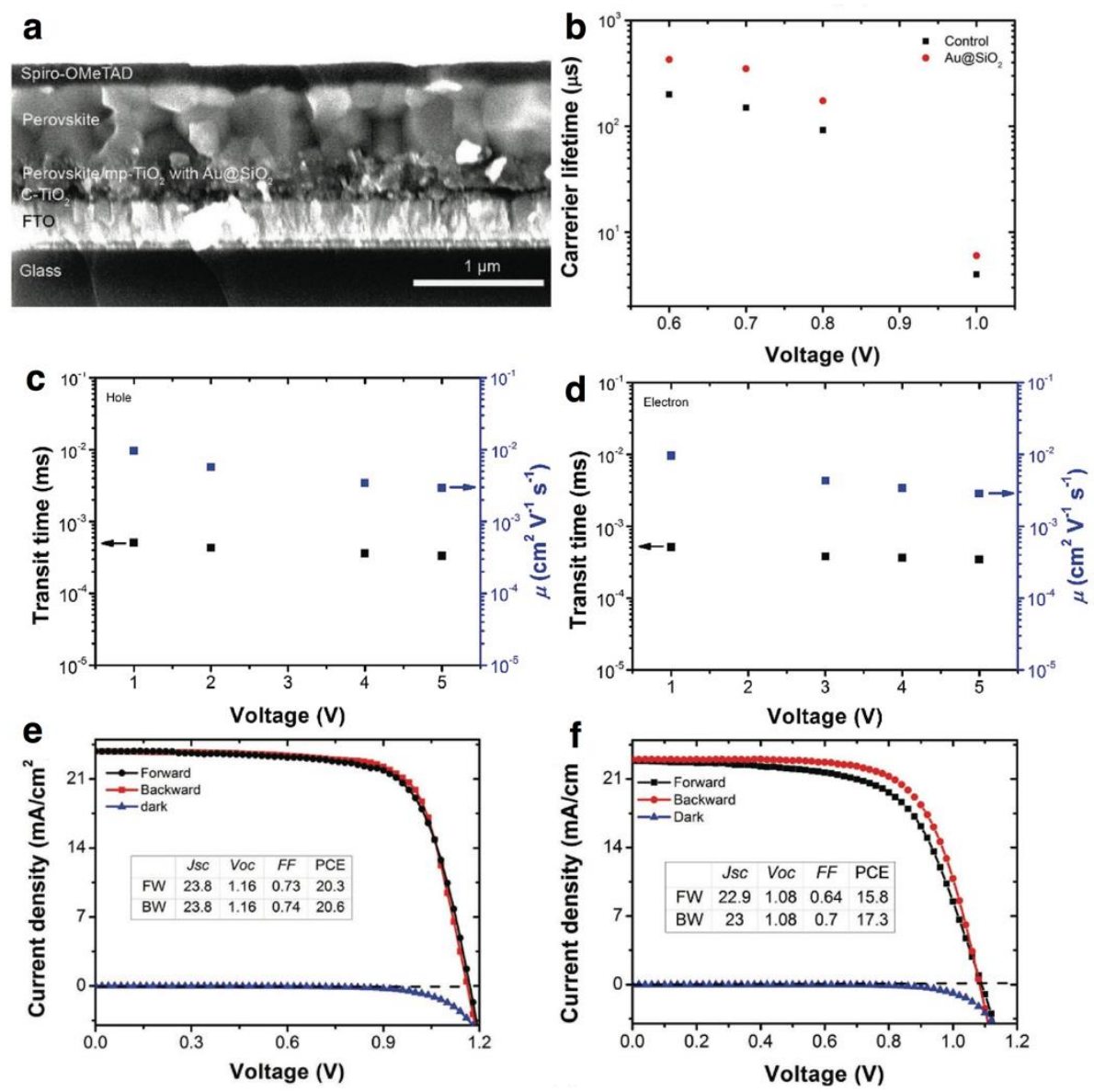

Figure 20. Prolonged charge carrier lifetimes and matching carrier mobility in plasmon enhanced mixed perovskite solar cells. a) Cross-sectional SEM image of a photovoltaic cell containing AuNSs with a diameter of about $40 \mathrm{~nm}$ coated with $15 \mathrm{~nm}$ of silica. b) Voltagedependent charge carrier lifetimes calculated from small perturbation transient photovoltage measurements for regular and plasmon-enhanced cells. The increased free carrier lifetime in the $\mathrm{Au} / \mathrm{SiO}_{2}$ containing cells translates into longer transport distances, leading to a higher PCE. c) Hole mobility and transit time of $\mathrm{Au} / \mathrm{SiO}_{2}$ containing devices, extracted from time of flight measurements. d) Corresponding electron mobility and transit time data. The match of the charge carrier mobilities in the $\mathrm{Au} / \mathrm{SiO}_{2}$ modified cells is remarkable and could explain the low hysteresis in the I-V scans shown in e) compared to the curves of the non-modified devices displayed in f). Adapted with permission. ${ }^{[187]}$ Copyright 2017, Wiley-VCH Verlag GmbH \& Co. KGaA, Weinheim.

\subsubsection{Enhancement Factors and Peak Performance}




\section{WILEY-VCH}

About one decade after the idea of plasmonic enhancement of thin film solar cells started to call the attention of materials scientists, ${ }^{[195]}$ it is nowadays clear that the incorporation of plasmonic core-shell particles can significantly improve PCEs, and typically reported enhanced factors of 10-50\% demonstrate that, with clever designs, detrimental effects such as parasitic light absorption and reduced conductivity are by far outweighed. ${ }^{[51,52,185]}$ For detailed lists of the achieved enhancement factors of different cell types the reader is referred to specific reviews. ${ }^{[184,185,196]}$ Intriguingly, besides a large volume of basic research and feasibility studies demonstrating impressive relative enhancements, recent publications on organic solar cells containing noble metal/ $\mathrm{SiO}_{2}$ particles with PCEs around 10\%, ${ }^{[51,197,198]}$ dye-sensitized cells with PCEs over $8 \%,{ }^{[92]}$ and perovskite cells with over $20 \%$ efficiency, ${ }^{[187]}$ have proven that plasmonic core-shell particles can be successfully integrated within high-performance thin film photovoltaics. Hereby, often energy conversion comparable to that of state of the art conventional designs has been achieved, albeit with lower material expenses. This development will not only foster the commercialization of lead-based perovskite photovoltaics, which have been under scrutiny for their potential environmental impact, but could ultimately lead to highly efficient novel solar cells approaching the Shockley-Queisser limit, with relatively simple configurations. ${ }^{[48]}$

\section{Conclusions}

The main conclusion of this review can be considered as a reminder that the combination of noble metals and silica is a highly effective and versatile approach to incorporate multiple functions within a single colloidal system. A variety of functions can be exerted by silica shells, as a function of their dimensions, degree of cross-linking, and porosity, including colloidal and chemical stabilization, spatial and electrical insulation, molecular sieving, loading of cargo, and even templating of more complex hybrid structures. Regarding the synthesis of silica-coated plasmonic particles, over two decades have passed since the first reports on noble metal/silica 


\section{WILEY-VCH}

nanocomposites, which have witnessed not only constant improvement of the classical Stöber method, but also the emergence of innovative procedures, such as the use of microemulsions, dry coating, or ultrasound- and microwave-assisted methods. This field has obviously reached sufficient maturity, leading to a high level of control over key chemical and morphological parameters as listed above. We can safely state that the degree of precision, reproducibility as well as cost and energy efficiency of the available procedures render silica coating a viable pathway to tailor nanoparticle properties on both the laboratory and industrial scales. As a consequence, a plethora of practical applications have been devised and implemented for such metallodielectric nanocomposites. Noble metal/silica core-shell particles and related derivatives opened new avenues toward applications that would just not be accessible using bare particles or soft coatings. Specifically, these functional colloids have facilitated the fabrication of refractive index and SERS sensors, multiplexed SERS tags, metal-enhanced fluorescent probes, theranostic and drug delivery systems, nanoparticle catalysts, and plasmonenhanced solar cells, among others.

An area in which we still see need for further investigations is the fundamental understanding of heteroparticle systems based on the localization of metallic and semiconducting materials at accurately defined distances, namely metal/quantum dot and metal/upconverting nanoparticle combinations. Furthermore, the incorporation of optically coupled nanoparticle assemblies, which offer strong near-field enhancement and exceptionally large scattering cross-sections, into diagnostic probes, photocatalysts, and solar cells, are worth a further scientific pursuit. We see a good opportunity regarding the use of tailored nanocomposites for chiral sensing, a field where surprisingly little has been reported. We should finally mention that the combination of noble metal nanoparticles and silica is not the only practical way to achieve the effects and applications described in this article. Other metal oxides, namely $\mathrm{SnO}_{2}, \mathrm{ZrO}_{2}, \mathrm{CuO}_{2}, \mathrm{CeO}_{2}$, and $\mathrm{TiO}_{2}$ have not only been shown to possess higher resistance against hydrolysis than $\mathrm{SiO}_{2}$, but 
can also introduce additional catalytic or electronic properties that could be exploited for further improvements in the performance of nanocomposite modified materials.

\section{Acknowledgements}

This work has been funded by the Spanish MINECO (Grants MAT2013-46101-R and MAT2017-86659-R). C. H. acknowledges the Alexander von Humboldt Foundation for funding within the framework of a Feodor Lynen fellowship.

Received: ((will be filled in by the editorial staff))

Revised: ((will be filled in by the editorial staff)) Published online: ((will be filled in by the editorial staff))

\section{References}

[1] Y. Sonnefraud, A. Leen Koh, D. W. McComb, S. A. Maier, Laser \& Photon. Rev. 2011, 6, 277.

[2] M. Grzelczak, J. Perez-Juste, P. Mulvaney, L. M. Liz-Marzan, Chem. Soc. Rev. 2008, 37, 1783.

[3] S. Liu, M. D. Regulacio, S. Y. Tee, Y. W. Khin, C. P. Teng, L. D. Koh, G. Guan, M.-Y. Han, Chem. Rec. 2016, 16, 1965.

[4] C. Hanske, G. González-Rubio, C. Hamon, P. Formentin, E. Modin, A. Chuvilin, A. Guerrero-Martínez, L. F. Marsal, L. M. Liz-Marzan, J. Phys. Chem. C 2017, 121, 10899.

[5] Q. Ruan, L. Shao, Y. Shu, J. Wang, H. Wu, Adv. Opt. Mater. 2013, 2, 65.

[6] L. Scarabelli, A. Sánchez-Iglesias, J. Perez-Juste, L. M. Liz-Marzán, J. Phys. Chem. Lett. 2015, 6, 4270.

[7] G. González-Rubio, P. Díaz-Núñez, A. Rivera, A. Prada, G. Tardajos, J. GonzálezIzquierdo, L. Bañares, P. Llombart, L. G. Macdowell, M. Alcolea Palafox, L. M. Liz-Marzan, O. Peña-Rodríguez, A. Guerrero-Martínez, Science 2017, 358, 640.

[8] L. Scarabelli, M. Coronado-Puchau, J. J. Giner-Casares, J. Langer, L. M. LizMarzán, ACS Nano 2014, 8, 5833.

[9] H. Yuan, C. G. Khoury, H. Hwang, C. M. Wilson, G. A. Grant, T. Vo-Dinh, Nanotechnol. 2012, 23, 075102.

[10] M. N. O’Brien, M. R. Jones, K. A. Brown, C. A. Mirkin, J. Am. Chem. Soc. 2014, 136, 7603.

[11] A. Tao, P. Sinsermsuksakul, P. Yang, Angew. Chem., Int. Ed. 2006, 45, 4597.

[12] S. Liu, M.-Y. Han, Chem. Asian J. 2010, 5, 36.

[13] A. Guerrero-Martínez, J. Perez-Juste, L. M. Liz-Marzan, Adv. Mater. 2010, 22, 1182.

[14] M. N. Sanz-Ortiz, K. Sentosun, S. Bals, L. M. Liz-Marzan, ACS Nano 2015, 9, 10489.

[15] J. Chen, R. Zhang, L. Han, B. Tu, D. Zhao, Nano Res. 2013, 6, 871.

[16] S. Biggs, P. Mulvaney, J. Chem. Phys. 1994, 100, 8501.

[17] J. L. Montaño-Priede, J. P. Coelho, A. Guerrero-Martínez, O. Peña-Rodríguez, U. Pal, J. Phys. Chem. C 2017, 121, 9543.

[18] M. P. Brandon, D. M. Ledwith, J. M. Kelly, J. Colloid Interface Sci. 2014, 415, 77. 
[19] T. Ung, L. M. Liz-Marzan, P. Mulvaney, J. Phys. Chem. B 2001, 105, 3441.

[20] A. Vanderkooy, Y. Chen, F. Gonzaga, M. A. Brook, ACS Appl. Mater. Interfaces 2011, 3, 3942.

[21] S. H. Joo, J. Y. Park, C.-K. Tsung, Y. Yamada, P. Yang, G. A. Somorjai, Nat. Mater. 2008, 8, 126.

[22] J.-N. Park, A. J. Forman, W. Tang, J. Cheng, Y.-S. Hu, H. Lin, E. W. McFarland, Small 2008, 4, 1694.

[23] J. C. Park, J. U. Bang, J. Lee, C. H. Ko, H. Song, J. Mater. Chem. 2010, 20, 1239.

[24] W. Albrecht, T.-S. Deng, B. Goris, M. A. van Huis, S. Bals, A. van Blaaderen, Nano Lett. 2016, 16, 1818.

[25] W. Albrecht, J. E. S. van der Hoeven, T.-S. Deng, P. E. de Jongh, A. van Blaaderen, Nanoscale 2017, 9, 2845.

[26] K. L. Wustholz, A.-I. Henry, J. M. McMahon, R. G. Freeman, N. Valley, M. E. Piotti, M. J. Natan, G. C. Schatz, R. P. Van Duyne, J. Am. Chem. Soc. 2010, 132, 10903.

[27] P. Botella, Í. Ortega, M. Quesada, R. F. Madrigal, C. Muniesa, A. Fimia, E. Fernández, A. Corma, Dalton Trans. 2012, 41, 9286.

[28] D. Rodríguez-Fernández, J. Langer, M. Henriksen-Lacey, L. M. Liz-Marzan, Chem. Mater. 2015, 27, 2540.

[29] M. Giersig, T. Ung, L. M. Liz-Marzan, P. Mulvaney, Adv. Mater. 1997, 9, 570.

[30] G. A. Sotiriou, T. Sannomiya, A. Teleki, F. Krumeich, J. Vörös, S. E. Pratsinis, Adv. Funct. Mater. 2010, 20, 4250.

[31] S. Shiomi, M. Kawamori, S. Yagi, E. Matsubara, J. Colloid Interface Sci. 2015, 460, 47.

[32] A. Wolcott, D. Gerion, M. Visconte, J. Sun, A. Schwartzberg, S. Chen, J. Z. Zhang, J. Phys. Chem. B 2006, 110, 5779.

[33] J. Rodríguez-Fernández, I. Pastoriza-Santos, J. Perez-Juste, F. J. García de Abajo, L. M. Liz-Marzan, J. Phys. Chem. C 2007, 111, 13361.

[34] H. Baida, P. Billaud, S. Marhaba, D. Christofilos, E. Cottancin, A. Crut, J. Lermé, P. Maioli, M. Pellarin, M. Broyer, N. Del Fatti, F. Vallée, A. Sánchez-Iglesias, I. Pastoriza-Santos, L. M. Liz-Marzán, Nano Lett. 2009, 9, 3463.

[35] V. Juvé, M. F. Cardinal, A. Lombardi, A. Crut, P. Maioli, J. Perez-Juste, L. M. LizMarzan, N. Del Fatti, F. Vallée, Nano Lett. 2013, 13, 2234.

[36] Y. R. Davletshin, A. Lombardi, M. F. Cardinal, V. Juvé, A. Crut, P. Maioli, L. M. Liz-Marzan, F. Vallée, N. D. Fatti, J. C. Kumaradas, ACS Nano 2012, 6, 8183.

[37] C. Hanske, M. Tebbe, C. Kuttner, V. Bieber, V. V. Tsukruk, M. Chanana, T. A. F. König, A. Fery, Nano Lett. 2014, 14, 6863.

[38] A. Lombardi, M. P. Grzelczak, E. Pertreux, A. Crut, P. Maioli, I. Pastoriza-Santos, L. M. Liz-Marzan, F. Vallée, N. Del Fatti, Nano Lett. 2016, 16, 6311.

[39] M. A. Noginov, G. Zhu, A. M. Belgrave, R. Bakker, V. M. Shalaev, E. E. Narimanov, S. Stout, E. Herz, T. Suteewong, U. Wiesner, Nature 2009, 460, 1110.

[40] X. Ma, H. Tan, T. Kipp, A. Mews, Nano Lett. 2010, 10, 4166.

[41] X. Ma, K. Fletcher, T. Kipp, M. P. Grzelczak, Z. Wang, A. Guerrero-Martínez, I. Pastoriza-Santos, A. Kornowski, L. M. Liz-Marzan, A. Mews, J. Phys. Chem. Lett. 2011, 2, 2466.

[42] N. S. Abadeer, M. R. Brennan, W. L. Wilson, C. J. Murphy, ACS Nano 2014, 8, 8392.

[43] H. Mishra, B. L. Mali, J. Karolin, A. I. Dragan, C. D. Geddes, Phys. Chem. Chem. Phys. 2013, 15, 19538.

[44] T. Ribeiro, C. Baleizão, J. P. S. Farinha, Sci. Rep. 2017, 7, 2440.

[45] J.-F. Li, Y. F. Huang, Y. Ding, Z. L. Yang, S. B. Li, X. S. Zhou, F. R. Fan, W. 
Zhang, Z. Y. Zhou, D. Y. Wu, B. Ren, Z. L. Wang, Z.-Q. Tian, Nature 2010, 464, 392.

[46] B.-Q. Zhang, S. B. Li, Q. Xiao, J. Li, J.-J. Sun, J. Raman Spectrosc. 2013, 44, 1120.

[47] X.-D. Tian, B.-J. Liu, J.-F. Li, Z. L. Yang, B. Ren, Z.-Q. Tian, J. Raman Spectrosc. 2013, 44, 994.

[48] W. Zhang, M. Saliba, S. D. Stranks, Y. Sun, X. Shi, U. Wiesner, H. J. Snaith, Nano Lett. 2013, 13, 4505.

[49] M. D. Brown, T. Suteewong, R. S. S. Kumar, V. D’Innocenzo, A. Petrozza, M. M. Lee, U. Wiesner, H. J. Snaith, Nano Lett. 2011, 11, 438.

[50] H. Choi, J.-P. Lee, S.-J. Ko, J.-W. Jung, H. Park, S. Yoo, O. Park, J.-R. Jeong, S. Park, J. Y. Kim, Nano Lett. 2013, 13, 2204.

[51] R. Zhang, Y. Zhou, L. Peng, X. Li, S. Chen, X. Feng, Y. Guan, W. Huang, Sci. Rep. 2016, 6, 25036.

[52] S. Carretero-Palacios, A. Jiménez-Solano, H. Míguez, ACS Energy Lett. 2016, 1, 323.

[53] Y. Wang, X. Zhou, C. Liang, P. Li, X. Hu, Q. Cai, Y. Zhang, F. Li, M. Li, Y. Song, Adv. Electron. Mater. 2017, 1700169.

[54] L. M. Liz-Marzan, M. Giersig, P. Mulvaney, Chem. Commun. 1996, 10, 731.

[55] K. Sentosun, M. N. Sanz-Ortiz, K. J. Batenburg, L. M. Liz-Marzan, S. Bals, Part. Part. Syst. Charact. 2015, 32, 1063.

[56] D. Rodríguez-Fernández, T. Altantzis, H. Heidari, S. Bals, L. M. Liz-Marzan, Chem. Commun. 2014, 50, 79.

[57] F. Wang, S. Cheng, Z. Bao, J. Wang, Angew. Chem., Int. Ed. 2013, 52, 10344.

[58] X. Zhu, H. Jia, X.-M. Zhu, S. Cheng, X. Zhuo, F. Qin, Z. Yang, J. Wang, Adv. Funct. Mater. 2017, 27, 1700016.

[59] G. Li, Z. Tang, Nanoscale 2014, 6, 3995.

[60] I. Pastoriza-Santos, J. Perez-Juste, L. M. Liz-Marzan, Chem. Mater. 2006, 18, 2465.

[61] Z. Lu, C. Gao, Q. Zhang, M. Chi, J. Y. Howe, Y. Yin, Nano Lett. 2011, 11, 3404.

[62] Q. Zeng, Y. Zhang, W. Ji, W. Ye, Y. Jiang, J. Song, ACS Appl. Mater. Interfaces 2014, 6, 19327.

[63] J. V. Jokerst, M. Thangaraj, P. J. Kempen, R. Sinclair, S. S. Gambhir, ACS Nano 2012, 6, 5920.

[64] Z. Zhou, C. Zhang, Q. Qian, J. Ma, P. Huang, X. zhang, L. Pan, G. Gao, H. Fu, S. Fu, H. Song, X. Zhi, J. Ni, D. Cui, J. Nanobiotechnol. 2013, 11, 17.

[65] H.-X. Xia, X.-Q. Yang, J.-T. Song, J. Chen, M.-Z. Zhang, D.-M. Yan, L. Zhang, M.-Y. Qin, L.-Y. Bai, Y.-D. Zhao, Z.-Y. Ma, J. Mater. Chem. B 2014, 2, 1945.

[66] J. Wang, R. Bai, R. Yang, J. Liu, J. Tang, Y. Liu, J. Li, Z. Chai, C. Chen, Metallomics 2015, 7, 516.

[67] C. Fernández-López, C. Mateo-Mateo, R. A. Alvarez-Puebla, J. Perez-Juste, I. Pastoriza-Santos, L. M. Liz-Marzan, Langmuir 2009, 25, 13894.

[68] M. Salehi, L. Schneider, P. Ströbel, A. Marx, J. Packeisen, S. Schlücker, Nanoscale 2014, 6, 2361.

[69] J. Rodríguez-Fernández, J. Perez-Juste, P. Mulvaney, L. M. Liz-Marzan, J. Phys. Chem. B 2005, 109, 14257.

[70] Z. Zhang, L. Wang, J. Wang, X. Jiang, X. Li, Z. Hu, Y. Ji, X. Wu, C. Chen, Adv. Mater. 2012, 24, 1418.

[71] J. Liu, C. Detrembleur, M.-C. De Pauw-Gillet, S. Mornet, C. Jérôme, E. Duguet, Small 2015, 11, 2323.

[72] J. Yang, D. Shen, L. Zhou, W. Li, X. Li, C. Yao, R. Wang, A. M. El-Toni, F. Zhang, D. Zhao, Chem. Mater. 2013, 25, 3030.

[73] Z. Jiang, B. Dong, B. Chen, J. Wang, L. Xu, S. Zhang, H. Song, Small 2013, 9, 604. 
[74] Z. Zhang, C. Liu, J. Bai, C. Wu, Y. Xiao, Y. Li, J. Zheng, R. Yang, W. Tan, ACS Appl. Mater. Interfaces 2015, 7, 6211.

[75] Z. Zhang, J. Wang, C. Chen, Adv. Mater. 2013, 25, 3869.

[76] M. A. Shenashen, S. A. El-Safty, E. A. Elshehy, J. Hazard. Mater. 2013, 260, 833.

[77] M. Khairy, S. A. El-Safty, M. A. Shenashen, E. A. Elshehy, Nanoscale 2013, 5, 7920.

[78] P. Piotrowski, J. Bukowska, J. Phys. Chem. C 2016, 120, 12092.

[79] C. Hamon, M. N. Sanz-Ortiz, E. Modin, E. H. Hill, L. Scarabelli, A. Chuvilin, L. M. Liz-Marzan, Nanoscale 2016, 8, 7914.

[80] G. Bodelón, V. Montes-García, V. López-Puente, E. H. Hill, C. Hamon, M. N. Sanz-Ortiz, S. Rodal-Cedeira, C. Costas, S. Celiksoy, I. Perez-Juste, L. Scarabelli, A. La Porta, J. Perez-Juste, I. Pastoriza-Santos, L. M. Liz-Marzan, Nat. Mater. 2016, 15, 1203.

[81] L. M. Liz-Marzan, A. P. Philipse, J. Colloid Interface Sci. 1995, 176, 459.

[82] L. M Liz-Marzan, P. Mulvaney, New J. Chem. 1998, 22, 1285.

[83] C. Graf, D. L. J. Vossen, A. Imhof, A. van Blaaderen, Langmuir 2003, 19, 6693.

[84] W. Stöber, A. Fink, E. Bohn, J. Colloid Interface Sci. 1968, 26, 62.

[85] L. M. Liz-Marzan, M. Giersig, P. Mulvaney, Langmuir 1996, 12, 4329.

[86] A. F. Wallace, J. J. DeYoreo, P. M. Dove, J. Am. Chem. Soc. 2009, 131, 5244.

[87] C. Xue, X. Chen, S. J. Hurst, C. A. Mirkin, Adv. Mater. 2007, 19, 4071.

[88] Y. J. Wong, L. Zhu, W. S. Teo, Y. W. Tan, Y. Yang, C. Wang, H. Chen, J. Am. Chem. Soc. 2011, 133, 11422.

[89] K. W. Shah, T. Sreethawong, S.-H. Liu, S.-Y. Zhang, L. S. Tan, M.-Y. Han, Nanoscale 2014, 6, 11273.

[90] M. Thiele, I. Götz, S. Trautmann, R. Müller, A. Csáki, T. Henkel, W. Fritzsche, Mater. Today 2015, 2, 33.

[91] Y. Zhang, X. Kong, B. Xue, Q. Zeng, X. Liu, L. Tu, K. Liu, H. Zhang, J. Mater. Chem. C 2013, 1, 6355.

[92] M. K. Gangishetty, K. E. Lee, R. W. J. Scott, T. L. Kelly, ACS Appl. Mater. Interfaces 2013, 5, 11044.

[93] T. Chen, G. Chen, S. Xing, T. Wu, H. Chen, Chem. Mater. 2010, 22, 3826.

[94] I. Pastoriza-Santos, L. M. Liz-Marzan, Langmuir 2002, 18, 2888.

[95] M. Grzelczak, J. Perez-Juste, P. Mulvaney, L. M. Liz-Marzan, Chem. Soc. Rev. 2008, 37, 1783.

[96] H. Sun, J. He, J. Wang, S.-Y. Zhang, C. Liu, T. Sritharan, S. Mhaisalkar, M.-Y. Han, D. Wang, H. Chen, J. Am. Chem. Soc. 2013, 135, 9099.

[97] G. P. Luke, A. Bashyam, K. A. Homan, S. Makhija, Y.-S. Chen, S. Y. Emelianov, Nanotechnol. 2013, 24, 455101.

[98] V. Salgueiriño-Maceira, M. A. Correa-Duarte, M. Spasova, L. M. Liz-Marzán, M. Farle, Adv. Funct. Mater. 2006, 16, 509.

[99] M. Sanles-Sobrido, W. Exner, L. Rodríguez-Lorenzo, B. Rodríguez-González, M. A. Correa-Duarte, R. A. Alvarez-Puebla, L. M. Liz-Marzan, J. Am. Chem. Soc. 2009, 131, 2699.

[100] F. J. Arriagada, K. Osseo-Asare, J. Colloid Interface Sci. 1999, 211, 210.

[101] C. Petit, P. Lixon, M. P. Pileni, J. Phys. Chem. 1993, 97, 12974.

[102] S. T. Selvan, T. T. Y. Tan, D. K. Yi, N. R. Jana, Langmuir 2010, 26, 11631.

[103] T. Li, J. Moon, A. A. Morrone, J. J. Mecholsky, D. R. Talham, J. H. Adair, Langmuir 1999, 15, 4328.

[104] S.-H. Wu, C.-T. Tseng, Y.-S. Lin, C.-H. Lin, Y. Hung, C.-Y. Mou, J. Mater. Chem. 2011, 21, 789.

[105] T. Ung, L. M. Liz-Marzan, P. Mulvaney, J. Phys. Chem. B 1999, 103, 6770. 
[106] J. Lee, J. C. Park, J. U. Bang, H. Song, Chem. Mater. 2008, 20, 5839.

[107] I. Gorelikov, N. Matsuura, Nano Lett. 2008, 8, 369.

[108] W.-C. Wu, J. B. Tracy, Chem. Mater. 2015, 27, 2888.

[109] J. Kobler, K. Möller, T. Bein, ACS Nano 2008, 2 , 791.

[110] V. Cauda, C. Argyo, D. G. Piercey, T. Bein, J. Am. Chem. Soc. 2011, 133, 6484.

[111] M. Broyer, S. Valange, J. P. Bellat, O. Bertrand, G. Weber, Z. Gabelica, Langmuir 2002, 18, 5083.

[112] Y. Hu, Y. Shi, H. Jiang, G. Huang, C. Li, ACS Appl. Mater. Interfaces 2013, 5, 10643.

[113] N. M. Bahadur, T. Furusawa, M. Sato, F. Kurayama, I. A. Siddiquey, N. Suzuki, J. Colloid Interface Sci. 2011, 355, 312.

[114] T. Zhang, H. Zhao, S. He, K. Liu, H. Liu, Y. Yin, C. Gao, ACS Nano 2014, 8, 7297.

[115] D. Zhao, J. Feng, Q. Huo, N. Melosh, G. Fredrickson, B. Chmelka, G. Stucky, Science 1998, 279, 548.

[116] Y.-J. Han, J. M. Kim, G. D. Stucky, Chem. Mater. 2000, 12, 2068.

[117] P. C. Angelomé, I. Pastoriza-Santos, J. Perez-Juste, B. Rodríguez-González, A. Zelcer, G. J. A. A. Soler-Illia, L. M. Liz-Marzan, Nanoscale 2012, 4, 931.

[118] M. Grzelczak, M. A. Correa-Duarte, L. M. Liz-Marzan, Small 2006, 2, 1174.

[119] M. Roca, A. J. Haes, J. Am. Chem. Soc. 2008, 130, 14273.

[120] R. J. Dillon, J.-B. Joo, F. Zaera, Y. Yin, C. J. Bardeen, Phys. Chem. Chem. Phys. 2013, 15, 1488.

[121] M. Coronado-Puchau, L. Saa, M. Grzelczak, V. Pavlov, L. M. Liz-Marzan, Nano Today 2013, 8, 461.

[122] L. Polavarapu, J. Perez-Juste, Q.-H. Xu, L. M. Liz-Marzan, J. Mater. Chem. C 2014, 2, 7460.

[123] B. N. Khlebtsov, V. A. Khanadeev, E. V. Panfilova, O. A. Inozemtseva, A. M. Burov, N. G. Khlebtsov, J. Quant. Spectrosc. Radiat. Transfer 2013, 121, 23.

[124] Y. Chen, X. Li, H. Zhou, X. Hong, Y. Geng, J. Phys. D: Appl. Phys. 2016, 1.

[125] I. Bruzas, S. Unser, S. Yazdi, E. Ringe, L. Sagle, Anal. Chem. 2016, 88, 7968.

[126] A. Tittl, X. Yin, H. Giessen, X.-D. Tian, Z.-Q. Tian, C. Kremers, D. N. Chigrin, N. Liu, Nano Lett. 2013, 13, 1816.

[127] S. Schlücker, Angew. Chem., Int. Ed. 2014, 53, 4756.

[128] K. A. Willets, R. P. Van Duyne, Annu. Rev. Phys. Chem. 2007, 58, 267.

[129] J. P. Camden, J. A. Dieringer, Y. Wang, D. J. Masiello, L. D. Marks, G. C. Schatz, R. P. Van Duyne, J. Am. Chem. Soc. 2008, 130, 12616.

[130] J. Langer, S. M. Novikov, L. M. Liz-Marzan, Nanotechnol. 2015, $26,1$.

[131] K. M. Mayer, J. H. Hafner, Chem. Rev. 2011, 111, 3828.

[132] J.-F. Li, S.-Y. Ding, Z. L. Yang, M.-L. Bai, J. R. Anema, X. Wang, A. Wang, D. Y. Wu, B. Ren, S.-M. Hou, T. Wandlowski, Z.-Q. Tian, J. Am. Chem. Soc. 2011, 133, 15922.

[133] C. Zheng, W. Shao, S. K. Paidi, B. Han, T. Fu, D. Wu, L. Bi, W. Xu, Z. Fan, I. Barman, Nanoscale 2015, 7, 16960.

[134] S. Carrasco, E. Benito-Peña, F. Navarro-Villoslada, J. Langer, M. N. Sanz-Ortiz, J. Reguera, L. M. Liz-Marzan, M. C. Moreno-Bondi, Chem. Mater. 2016, $28,7947$.

[135] A. Shiohara, Y. Wang, L. M. Liz-Marzan, J. Photochem. Photobiol. C 2014, 21, 2.

[136] S. R. Panikkanvalappil, M. A. El-Sayed, J. Phys. Chem. B 2014, 118, 14085.

[137] C. Hamon, M. N. Sanz-Ortiz, E. Modin, E. H. Hill, L. Scarabelli, A. Chuvilin, L. M. Liz-Marzan, Nanoscale 2016, 8, 7914.

[138] W. Liu, Z. Zhu, K. Deng, Z. Li, Y. Zhou, H. Qiu, Y. Gao, S. Che, Z. Tang, J. Am. Chem. Soc. 2013, 135, 9659.

[139] M. Thomas, J. J. Greffet, R. Carminati, J. R. Arias-Gonzalez, Appl. Phys. Lett. 
2004, 85, 3863.

[140] R. Bardhan, N. K. Grady, N. J. Halas, Small 2008, 4, 1716.

[141] P. Anger, P. Bharadwaj, L. Novotny, Phys. Rev. Lett. 2006, 96, 113002.

[142] Y. Teng, K. Ueno, X. Shi, D. Aoyo, J. Qiu, H. Misawa, Ann. Phys. 2012, 524, 733.

[143] J. M. Blake-Hedges, S. H. Greenspan, J. A. Kean, M. A. McCarron, M. L. Mendonca, K. L. Wustholz, Chem. Phys. Lett. 2015, 635, 328.

[144] G. Zheng, J. Wang, L. Kong, H. Cheng, J. Liu, Plasmonics 2012, 7, 487.

[145] C. Niu, Q. Song, G. He, N. Na, J. Ouyang, Anal. Chem. 2016, 88, 11062.

[146] M.-K. Nguyen, W.-N. Su, B.-J. Hwang, Adv. Healthcare Mater. 2017, 6, 1601290.

[147] E.-K. Lim, T. Kim, S. Paik, S. Haam, Y.-M. Huh, K. Lee, Chem. Rev. 2014, 115, 327.

[148] L. C. Kennedy, L. R. Bickford, N. A. Lewinski, A. J. Coughlin, Y. Hu, E. S. Day, J. L. West, R. A. Drezek, Small 2011, 7, 169.

[149] A. M. Smith, M. C. Mancini, S. Nie, Nat. Nanotechnol. 2009, 4, 710 EP.

[150] R. Bardhan, N. K. Grady, J. R. Cole, A. Joshi, N. J. Halas, ACS Nano 2009, 3, 744.

[151] L. Rodríguez-Lorenzo, Z. Krpetic, S. Barbosa, R. A. Alvarez-Puebla, L. M. LizMarzan, I. A. Prior, M. Brust, Integr. Biol. 2011, 3, 922.

[152] X. Xia, W. Li, Y. Zhang, Y. Xia, Interface Focus 2013, 3, 20120092.

[153] D. Jimenez de Aberasturi, A. B. Serrano-Montes, J. Langer, M. Henriksen-Lacey, W. J. Parak, L. M. Liz-Marzan, Chem. Mater. 2016, 28, 6779.

[154] S. Harmsen, M. A. Wall, R. Huang, M. F. Kircher, Nat. Protoc. 2017, 12, 1400.

[155] Z. Wang, S. Zong, H. Chen, C. Wang, S. Xu, Y. Cui, Adv. Healthcare Mater. 2014, 3, 1889.

[156] Y.-S. Chen, W. Frey, S. Kim, P. Kruizinga, K. Homan, S. Emelianov, Nano Lett. 2011, 11, 348 .

[157] P. Sharma, S. C. Brown, N. Bengtsson, Q. Zhang, G. A. Walter, S. R. Grobmyer, S. Santra, H. Jiang, E. W. Scott, B. M. Moudgil, Chem. Mater. 2008, $20,6087$.

[158] M. Hembury, C. Chiappini, S. Bertazzo, T. L. Kalber, G. L. Drisko, O. Ogunlade, S. Walker-Samuel, K. S. Krishna, C. Jumeaux, P. Beard, C. S. S. R. Kumar, A. E. Porter, M. F. Lythgoe, C. Boissière, C. Sanchez, M. M. Stevens, Proc. Natl. Acad. Sci. U. S. A. 2015, 112, 1959.

[159] N. S. Abadeer, C. J. Murphy, J. Phys. Chem. C 2016, 120, 4691.

[160] R. Bardhan, S. Lal, A. Joshi, N. J. Halas, Acc. Chem. Res. 2011, 44, 936.

[161] H. Petrova, J. Perez-Juste, I. Pastoriza-Santos, G. V. Hartland, L. M. Liz-Marzan, P. Mulvaney, Phys. Chem. Chem. Phys. 2006, 8, 814.

[162] C.-W. Chen, P.-H. Lee, Y.-C. Chan, M. Hsiao, C.-H. Chen, P. C. Wu, P. R. Wu, D. P. Tsai, D. Tu, X. Chen, R.-S. Liu, J. Mater. Chem. B 2015, 3, 8293.

[163] S. Rohani, M. Quintanilla, S. Tuccio, F. De Angelis, E. Cantelar, A. O. Govorov, L. Razzari, F. Vetrone, Adv. Opt. Mater. 2015, 3, 1606.

[164] S. Mura, J. Nicolas, P. Couvreur, Nat. Mater. 2013, 12, 991.

[165] M. Vallet-Regí, F. Balas, D. Arcos, Angew. Chem., Int. Ed. 2007, 46, 7548.

[166] I. Urries, C. Muñoz, L. Gomez, C. Marquina, V. Sebastian, M. Arruebo, J. Santamaría, Nanoscale 2014, 6, 9230.

[167] C. Andreou, V. Neuschmelting, D.-F. Tschaharganeh, C.-H. Huang, A. Oseledchyk, P. Iacono, H. Karabeber, R. R. Colen, L. Mannelli, S. W. Lowe, M. F. Kircher, ACS Nano 2016, 10, 5015.

[168] R. Bazak, M. Houri, S. El Achy, S. Kamel, T. Refaat, J. Cancer Res. Clin. Oncol. 2015, 141, 769 .

[169] S. S. Lucky, K. C. Soo, Y. Zhang, Chem Rev 2015, 115, 1990.

[170] D. Astruc, F. Lu, J. R. Aranzaes, Angew. Chem., Int. Ed. 2005, 44, 7852.

[171] S. Wang, M. Zhang, W. Zhang, ACS Catal. 2011, 1, 207. 
[172] J. Lee, J. C. Park, H. Song, Adv. Mater. 2008, 20, 1523.

[173] S. Liu, S.-Q. Bai, Y. Zheng, K. W. Shah, M.-Y. Han, ChemCatChem 2012, 4, 1462.

[174] Y. Ge, T. Gao, C. Wang, Z. H. Shah, R. Lu, S. Zhang, J. Colloid Interface Sci. 2017, 491, 123.

[175] J. Ge, Q. Zhang, T. Zhang, Y. Yin, Angew. Chem. 2008, 120, 9056.

[176] Q. Zhang, T. Zhang, J. Ge, Y. Yin, Nano Lett. 2008, 8, 2867.

[177] S. Liu, Z. Zhang, M. Y. Han, Adv. Mater. 2005, 17, 1862.

[178] S. Soulé, A.-L. Bulteau, S. Faucher, B. Haye, C. Aimé, J. Allouche, J.-C. Dupin, G. Lespes, T. Coradin, H. Martinez, Langmuir 2016, 32, 10073.

[179] M. Sanles-Sobrido, M. Pérez-Lorenzo, B. Rodríguez-González, V. Salgueiriño, M. A. Correa-Duarte, Angew. Chem., Int. Ed. 2012, 51, 3877.

[180] R. L. Oliveira, P. K. Kiyohara, L. M. Rossi, Green Chem. 2010, 12, 144.

[181] M. J. Jacinto, P. K. Kiyohara, S. H. Masunaga, R. F. Jardim, L. M. Rossi, Appl. Catal., A 2008, 338, 52.

[182] T. Torimoto, H. Horibe, T. Kameyama, K.-I. Okazaki, S. Ikeda, M. Matsumura, A. Ishikawa, H. Ishihara, J. Phys. Chem. Lett. 2011, 2, 2057.

[183] H. A. Atwater, A. Polman, Nat. Mater. 2010, 9, 205.

[184] M. Notarianni, K. Vernon, A. Chou, M. Aljada, J. Liu, N. Motta, Sol. Energy 2014, 106, 23.

[185] W. R. Erwin, H. F. Zarick, E. M. Talbert, R. Bardhan, Energy Environ. Sci. 2016, 9, 1577.

[186] V. Janković, Y. M. Yang, J. You, L. Dou, Y. Liu, P. Cheung, J. P. Chang, Y. Yang, ACS Nano 2013, 7, 3815.

[187] T. Ye, S. Ma, X. Jiang, L. Wei, C. Vijila, S. Ramakrishna, Adv. Funct. Mater. 2017, 27, 1606545.

[188] Y. H. Jang, Y. J. Jang, S. Kim, L. N. Quan, K. Chung, D. H. Kim, Chem Rev 2016, 116, 14982.

[189] J.-Y. Lee, P. Peumans, Opt. Express 2010, 18, 10078.

[190] K. Yao, X.-K. Xin, C.-C. Chueh, K.-S. Chen, Y.-X. Xu, A. K. Y. Jen, Adv. Funct. Mater. 2015, 25, 567.

[191] J. Li, S. K. Cushing, F. Meng, T. R. Senty, A. D. Bristow, N. Wu, Nat. Photonics 2015, 9, 601.

[192] B. Y. Zheng, H. Zhao, A. Manjavacas, M. McClain, P. Nordlander, N. J. Halas, Nat. Commun. 2015, 6, 7797.

[193] A. Manjavacas, J. G. Liu, V. Kulkarni, P. Nordlander, ACS Nano 2014, 8, 7630.

[194] R. Wu, B. Yang, C. Zhang, Y. Huang, Y. Cui, P. Liu, C. Zhou, Y. Hao, Y. Gao, J. Yang, J. Phys. Chem. C 2016, 120, 6996.

[195] K. R. Catchpole, A. Polman, Opt. Express 2008, 16, 21793.

[196] D. Thrithamarassery Gangadharan, Z. Xu, Y. Liu, R. Izquierdo, D. Ma, Nanophotonics 2017, 6, 111.

[197] S. Liu, R. Jiang, P. You, X. Zhu, J. Wang, F. Yan, Energy Environ. Sci. 2016, 9, 898.

[198] H. I. Park, S. Lee, J. M. Lee, S. A. Nam, T. Jeon, S. W. Han, S. O. Kim, ACS Nano 2014, 8, 10305.

Table of Contents Material

Silica-Coated Plasmonic Metal Nanoparticles in Action

C. Hanske,* M. N. Sanz-Ortiz, L. M. Liz-Marzán* 


\section{WILEY-VCH}

Metal/silica nanocomposites have been under intensive investigation for over 20 years. Besides the development of a wide array of synthesis strategies for the preparation of highly regular hybrid plasmonic nanoparticles, a considerable number of practical applications have been presented in sensing, theranostics, catalysis, and photovoltaics. Herein, an overview of the recent advances in the design and utilization of such materials is provided.

Keywords: core-shell colloids, plasmonic sensing, theranostics, nanoparticle catalysis, plasmon-enhanced solar cells

\section{ToC figure}

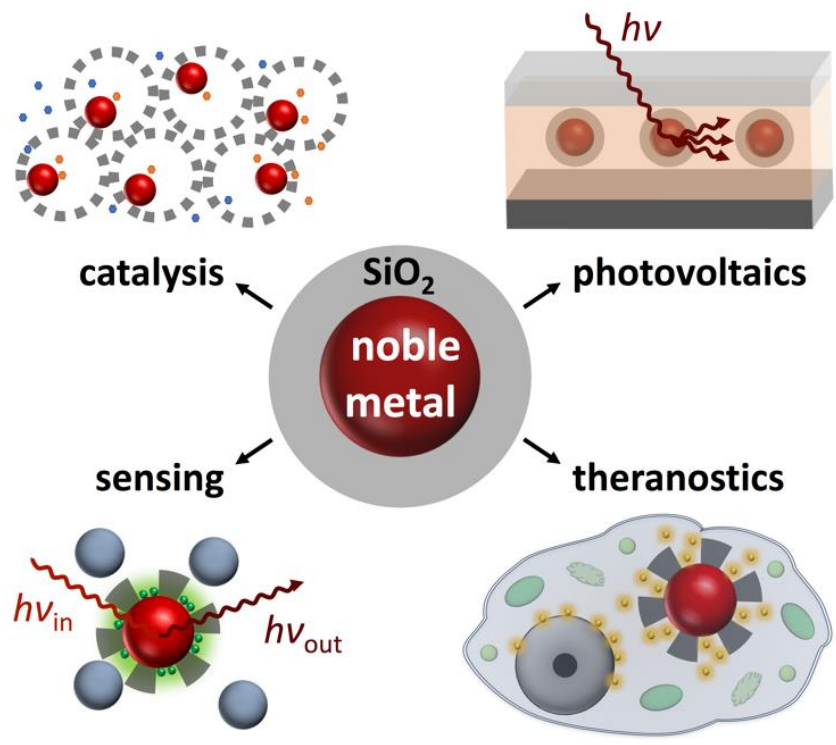

Copyright WILEY-VCH Verlag GmbH \& Co. KGaA, 69469 Weinheim, Germany, 2018. 